ARTICLE

\title{
Targeting aberrant DNA methylation in mesenchymal stromal cells as a treatment for myeloma bone disease
}

\author{
Antonio Garcia-Gomez 1,2,11凶 ${ }^{\text {, Tianlu Li1,2,11 }}$, Carlos de la Calle-Fabregat ${ }^{1,2}$, Javier Rodríguez-Ubreva (i) ${ }^{1,2}$, \\ Laura Ciudad ${ }^{1,2}$, Francesc Català-Moll1,2, Gerard Godoy-Tena ${ }^{1}$, Montserrat Martín-Sánchez ${ }^{3}$, \\ Laura San-Segundo ${ }^{3}$, Sandra Muntión ${ }^{3}$, Xabier Morales (10 4, Carlos Ortiz-de-Solórzano (1) 4, Julen Oyarzabal ${ }^{5}$, \\ Edurne San José-Enériz ${ }^{6}$, Manel Esteller (i) ${ }^{7,8,9,10}$, Xabier Agirre (iD) ${ }^{6}$, Felipe Prosper (D) ${ }^{6}$, Mercedes Garayoa (iD ${ }^{3}$ \& \\ Esteban Ballestar (1D) 1,2凶
}

Multiple myeloma (MM) progression and myeloma-associated bone disease (MBD) are highly dependent on bone marrow mesenchymal stromal cells (MSCs). MM-MSCs exhibit abnormal transcriptomes, suggesting the involvement of epigenetic mechanisms governing their tumor-promoting functions and prolonged osteoblast suppression. Here, we identify widespread DNA methylation alterations of bone marrow-isolated MSCs from distinct MM stages, particularly in Homeobox genes involved in osteogenic differentiation that associate with their aberrant expression. Moreover, these DNA methylation changes are recapitulated in vitro by exposing MSCs from healthy individuals to MM cells. Pharmacological targeting of DNMTs and G9a with dual inhibitor CM-272 reverts the expression of hypermethylated osteogenic regulators and promotes osteoblast differentiation of myeloma MSCs. Most importantly, CM-272 treatment prevents tumor-associated bone loss and reduces tumor burden in a murine myeloma model. Our results demonstrate that epigenetic aberrancies mediate the impairment of bone formation in $M M$, and its targeting by $\mathrm{CM}-272$ is able to reverse $M B D$.

\footnotetext{
${ }^{1}$ Epigenetics and Immune Disease Group, Josep Carreras Leukaemia Research Institute (IJC), Badalona, 08916 Badalona, Barcelona, Spain. ${ }^{2}$ Chromatin and Disease Group, Cancer Epigenetics and Biology Programme (PEBC), Bellvitge Biomedical Research Institute (IDIBELL), 08908 L'Hospitalet de Llobregat, Barcelona, Spain. ${ }^{3}$ Centro de Investigación del Cáncer, IBMCC (Universidad de Salamanca-CSIC) and Hospital Universitario de Salamanca-IBSAL, 37007 Salamanca, Spain. ${ }^{4}$ Imaging Platform, Center for Applied Medical Research (CIMA), University of Navarra, IDISNA, Ciberonc, 31008 Pamplona, Spain. ${ }_{5}^{5}$ Small Molecule Discovery Platform, Molecular Therapeutics Program, Center for Applied Medical Research (CIMA), University of Navarra, 31008 Pamplona, Spain. ${ }^{6}$ Division of Hemato-Oncology, Center for Applied Medical Research (CIMA), University of Navarra, IDISNA, Ciberonc 31008 Pamplona, Spain. ${ }^{7}$ Josep Carreras Leukaemia Research Institute (IJC), Badalona, Barcelona, Catalonia, Spain. ${ }^{8}$ Centro de Investigacion Biomedica en Red Cancer (CIBERONC), Madrid, Spain. ${ }^{9}$ Institucio Catalana de Recerca i Estudis Avançats (ICREA), Barcelona, Catalonia, Spain. ${ }^{10}$ Physiological Sciences Department, School of Medicine and Health Sciences, University of Barcelona (UB), Barcelona, Catalonia, Spain. ${ }^{11}$ These authors contributed equally: Antonio Garcia-

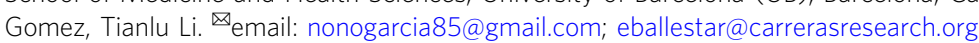


$\mathrm{M}$ ultiple myeloma (MM) is an incurable hematological malignancy characterized by clonal expansion of plasma cells in the bone marrow (BM) that accounts for $1 \%$ of all cancers 1,2 . Nearly $90 \%$ of myeloma patients suffer from skeletal-related events during the course of the disease, including severe bone pain, hypercalcemia, pathological fractures, and spinal cord compression ${ }^{3}$, that not only affect the quality of life but also their overall survival ${ }^{4}$. Myelomaassociated bone disease (MBD) is characterized by an increase in bone-resorptive activity and number of osteoclasts (OCs), as well as impairment of bone-forming activity and differentiation of osteoblasts $(\mathrm{OBs})$, which ultimately lead to the development of osteolytic lesions 5 .

In most cases, symptomatic myeloma is preceded by sequential asymptomatic stages of monoclonal gammopathy of undetermined significance (MGUS) and smoldering myeloma (SMM), with increasing BM plasmocytosis and monoclonal component as well as augmented risk of progression to active $\mathrm{MM}^{6,7}$. The biological behavior and clinical outcome of $\mathrm{MM}$ are partly dependent on genetic and epigenetic abnormalities of tumor subclones that arise from MGUS and SMM stages ${ }^{8}$. However, the clinical stability of MGUS cases, despite displaying shared genetic lesions with MM cells, suggests that the BM microenvironment may critically modulate disease progression ${ }^{6,9,10}$. In this regard, it has been widely shown that a complex and bidirectional relationship exists between MM cells and the BM niche, which results in oncogenesis support, anemia, immunosuppression, and uncoupling of the bone remodeling process ${ }^{11}$.

Mesenchymal stromal cells (MSCs) are an essential cell type in the formation and function of the BM microenvironment, being the progenitors of bone-forming OBs, adipocytes, and chondroblasts, as well as the hematopoietic-supporting stroma components of the $\mathrm{BM}^{12}$. It is well-documented that BMderived MSCs from MM patients contribute to MM progression (reviewed in ref. ${ }^{11}$ ) and show an impaired ability to differentiate into $\mathrm{OBs}^{13,14}$. Moreover, MM-MSCs are considered inherently abnormal, as their dysfunctionality remains even following ex vivo culture in the absence of MM cells ${ }^{15}$. Furthermore, bone lesions persist in many MM patients even after therapeutic remission, suggesting a long-term defect in MSCs that inhibit their ability to properly differentiate into functional $\mathrm{OBs}^{16}$

Previous studies described that MSCs from MM patients are cytogenetically normal ${ }^{17,18}$, but show alterations in their transcriptional ${ }^{13,19}$ and proteomic ${ }^{11}$ profiles even in the absence of myeloma cell interaction. This suggests that epigenetic mechanisms could be governing the tumor-promoting functions of MSCs and their prolonged OB suppression in MM. In fact, Adamik and colleagues ${ }^{20}$ reported abnormal recruitment of chromatin remodelers in MSCs from myeloma patients, contributing to the transcriptional repression of Runx2, a master regulator of $\mathrm{OB}$ differentiation. Yet, there is a lack of information about DNA methylation-related mechanisms that may contribute to MM progression and subsequent bone defects. DNA methylation is an essential epigenetic modification involving the addition of a methyl group to the 5-carbon of the cytosine ring by a family of DNA methyltransferase (DNMT) enzymes ${ }^{21}$, which has been described to play a critical role in MSC lineage determination $^{22}$, as well as in tumor progression and immunosuppression in other cancer types ${ }^{23}$.

In this study, we identify DNA methylation alterations in MSCs of MM patients mediated by MM cells resulting in the dysregulation of osteogenesis, and this is reversed by the treatment with CM-272, a dual inhibitor of DNMTs and the histone methyltransferase G9a.

\section{Results}

BM-derived MSCs of distinct MM stages exhibit altered DNA methylation profiles. We first obtained genome-wide DNA methylation profiles of BM-derived MSCs isolated at different stages of MM (newly diagnosed MGUS, high-risk SMM, and $\mathrm{MM}$ ) and healthy controls. DNA methylation changes were identified using two different statistical approaches (Fig. 1A): (i) detection of differentially methylated CpG positions (DMPs) based on differences in DNA methylation means between the patient (MGUS, SMM, and MM) and healthy MSCs $(\Delta \beta \geq 0.1$ and $*^{*} p<0.01$ ) (Supplementary Data 1 ); and (ii) detection of differentially variable CpG positions (DVPs) based on differences in variance of DNA methylation levels $\left(q \mathrm{val}<0.05\right.$ and $\left.*^{*}<0.05\right)$ between the sample groups (Supplementary Data 2). In regards to DMPs, the largest number of altered CpGs was found in MSCs from patients of the SMM stage compared to healthy donors (Supplementary Fig. 1A, B). On the other hand, we observed the highest number of DVPs in comparison to healthy donors in MSCs isolated from MGUS followed by SMM and MM patients (Supplementary Fig. 1C, D), supporting the notion that these stochastic and heterogeneous DNA methylation patterns are associated with early stages of carcinogenesis, as previously reported $^{24,25}$. We also observed that the majority of identified DMPs and DVPs are disease stage-specific, although the asymptomatic stages showed a moderate proportion of overlap (Fig. 1A).

Given that myeloma is a multi-stage disease, we then analyzed the accumulative changes of DNA methylation associated with MM progression by selecting DMPs (Supplementary Data 3) and DVPs (Supplementary Data 4) that were found either only in the MM stage, shared by SMM and MM and in all three stages (Fig. 1A). With this analysis, we identified 872 hyperand 951 hypomethylated DMPs, and 260 hyper- and 318 hypomethylated DVPs.

Analyzing the distribution of MM progression-associated CpGs in relation to $\mathrm{CpG}$ islands (CGI), we observed a significant enrichment of $\mathrm{CpGs}$ in open sea regions in the hypomethylated DMP data set (Fig. 1B) and in CpG islands in the hypermethylated DVP data set (Supplementary Fig. 1E). Utilizing publicly available chromatin state maps of BM-derived MSCs from healthy individuals ${ }^{26}$, we found a significant enrichment of both hyper- and hypomethylated DMPs sites that correspond to enhancers (Fig. 1C). In addition, we observed an enrichment in flanking transcription start sites (TSS) in the hypomethylated set, and regions repressed by Polycomb Group (PcG) in hypermethylated CpGs (Fig. 1C). On the other hand, hypermethylated DVPs were enriched in TSSs, bivalent regions, and regions repressed by Polycomb (Supplementary Fig. 1F).

To determine whether these MM progression-associated loci shared any common DNA elements, we performed a search for enriched transcription factor (TF)-binding sites in these regions using the HOMER algorithm ${ }^{27}$. We observed a significant overrepresentation of binding sites for the Runt and Tead family in differentially hypermethylated DMPs associated with MM progression $(* * p<0.01$; Fig. $1 \mathrm{D})$. These results suggest that key transcription factors involved in the upregulation of osteogenic genes, such as $R U N X 2^{28}$ or TEAD $2^{29}$, may participate in aberrant DNA hypermethylation. Since DNA methylation has been originally linked to transcriptional repression, these results suggested that the hypermethylation of these regions could compromise the ability of MSCs to undergo proper OB differentiation. On the other hand, DMP sites that experienced aberrant DNA hypomethylation were highly enriched in binding motifs of the bZip and Homeobox families $\left({ }^{* *} p<0.01\right.$; Fig. 1D). In this respect, the loss of DNA methylation could be selectively driving the occupancy of TF 
A

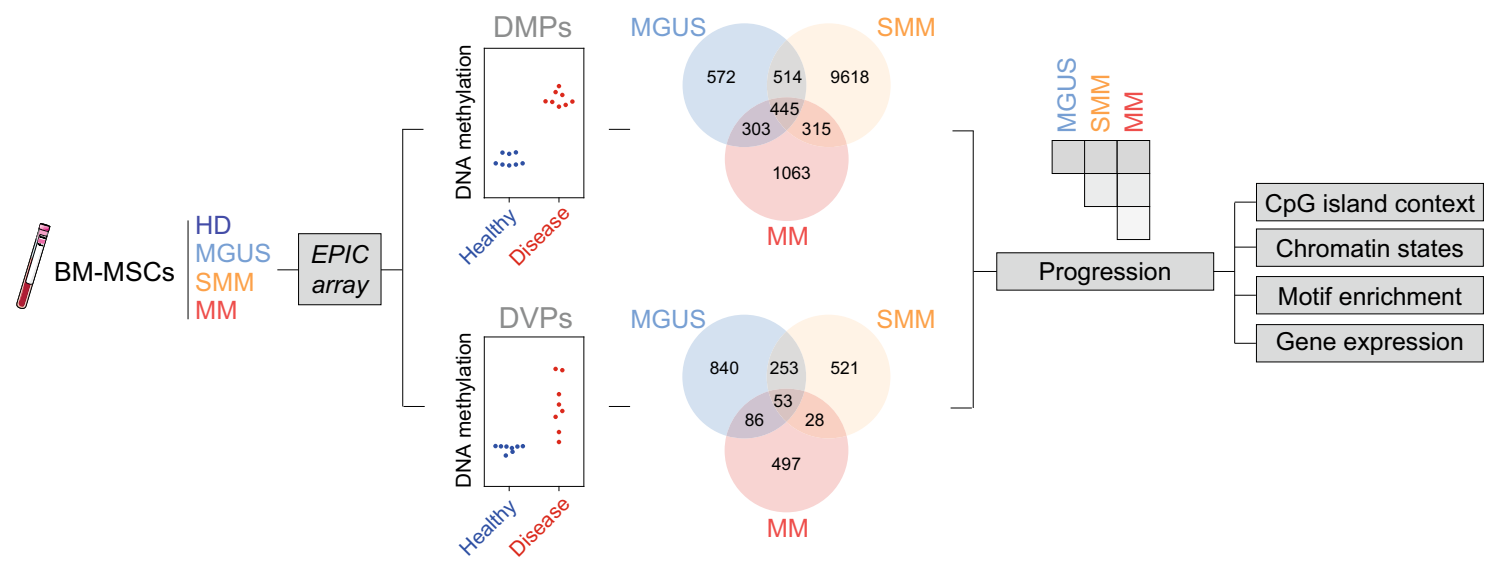

B

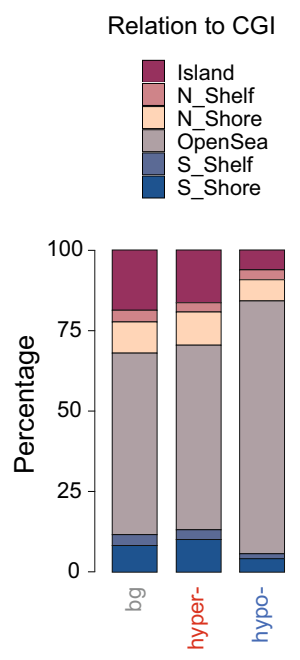

C

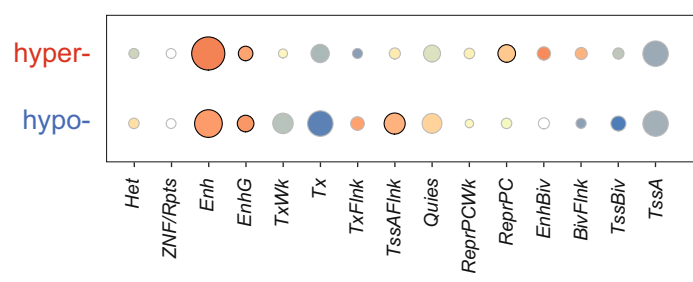

$\log$ (odd ratio)

FDR Significant

$\bigcirc_{1}^{\circ} 1$ e- $\bigcirc$ No

$\bigcirc^{1 e-12}{ }^{1 e-06}$

$\bigcirc_{1 \mathrm{e}-18}$

$\bigcirc_{1 e-24}$
D

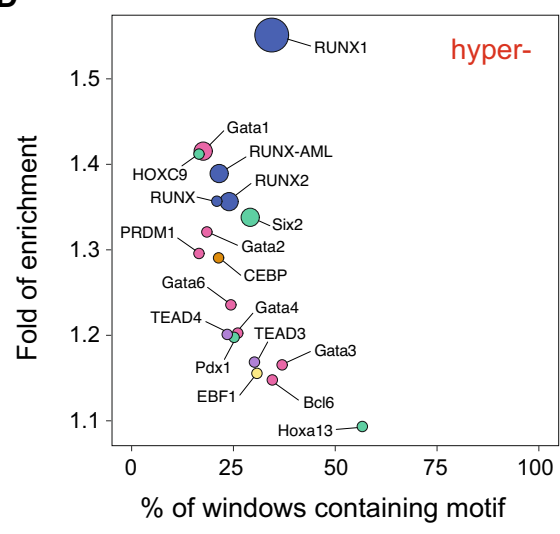

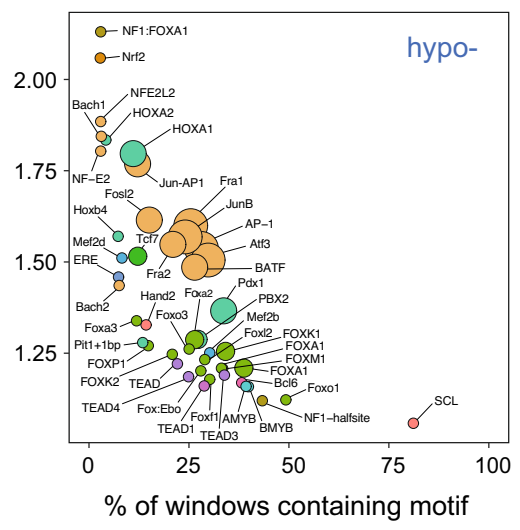

TF family

$\circ \mathrm{bHLH}$
$\circ \mathrm{bZIP}$

CTF

$O$ EBF
$O$ Forkhea

HMG

O Homeobox

HTH

MADS

Runt

TEA

TEA
TEAD
Zf

$\mathrm{p}$ value

- 0.05 to $1 \mathrm{e}-03$

- $1 \mathrm{e}-03$ to $1 \mathrm{e}-05$

$1 \mathrm{e}-05$ to $1 \mathrm{e}-08$

E
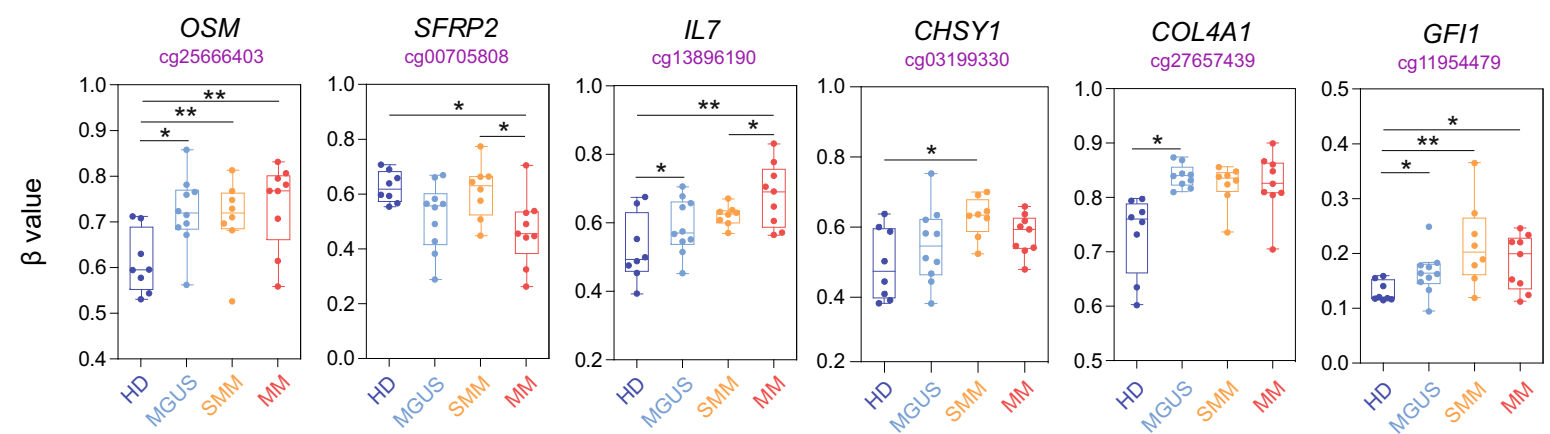

that have been reported as negative regulators of $\mathrm{OB}$ differentiation such as HOXA $2^{30}$ and $\mathrm{ATF} 3^{31}$. In addition, we observed transcriptional deregulation of some members of these TF families using expression array data from BM-derived MSCs of healthy controls, MGUS, SMM, and MM patients. Some of these TFs were specifically downregulated in MSCs of active myeloma (RUNX2 and TEAD2), others were already downregulated in precursor myeloma stages (HOXC9 and CEBPD), whereas other TFs, including HOXA2 and ATF3, did not change their expression in any myeloma stages
(Supplementary Fig. 1G). In all, these findings suggested that MM progression-associated DNA methylation changes in MSCs might be mediated by the sequential activity of specific TF families, which are also functionally deregulated in $\mathrm{MM}^{32}$. Furthermore, other genes that play important roles in the pathophysiology of MM (such as the cytokines IL6 and OSM) and associated MBD (secreted factors such as RANKL, SFRP2, IL7, CHSY1, COL4A1, and the transcriptional repressor GFI1) were also found to alter their DNA methylation levels (Fig. 1E and Supplementary Fig. 1H). 
Fig. 1 High-throughput stepwise DNA methylation changes in BM-derived MSCs associated with MM progression. A Workflow depicting the methodological approach for selecting DNA methylation changes in bone marrow-derived mesenchymal stromal cells (BM-MSCs) from monoclonal gammopathy of undetermined significance (MGUS; $n=10)$, smoldering myeloma (SMM; $n=8)$, and multiple myeloma $(M M ; n=9)$ patients versus healthy controls $(H D ; n=8)$. An example of a $C p G$ site experiencing increased mean (differentially methylated position, DMP) or variance (differentially variable position, DVP) in the disease versus the control condition is shown. Venn diagrams show the number of DMPs or DVPs resulting from each comparison. B Distribution of DNA methylation changes in relation to CpG islands (CGI), including shores (south, S; north, N), shelves (south, S; north, N), and open sea regions for differentially hyper- or hypomethylated $\mathrm{CpG}$ sites. C Enrichment analysis of differentially hyper-and hypomethylated $\mathrm{CpG}$ sites located in different genomic regions, annotated by 15 chromHMM states. Color scale refers to log odd ratio and circle size refers to $p$-value significance. D Bubble plot representation of HOMER transcription factor (TF) motif enrichment analysis of differentially hyper- and hypomethylated CpGs in MSCs during MM progression (left and right panel, respectively). Color range depicts different transcription factor families and circle size refers to $p$-value significance. E Box plots showing $\beta$-values from DMPs obtained from the EPIC array in MSCs from healthy donors and MGUS, SMM, and MM patients of relevant genes involved in the pathogenesis of $M M$ and associated bone disease. HD is represented in dark blue, MGUS in light blue, SMM in orange, and MM in red. eBayes-moderated ANOVA $t$-test was performed to calculate statistical significance $\left({ }^{\star} p<0.05,{ }^{\star \star} p<0.01\right.$, and $\left.{ }^{\star \star \star} p<0.005\right)$.

\begin{abstract}
Aberrant DNA methylation is associated with differential Homeobox gene expression in MSCs at different MM stages. To further investigate the relationship between differential DNA methylation and gene expression, we mapped the DMPs to the most proximal gene. Using expression array data from BMderived MSCs of healthy controls, MGUS, SMM, and MM patients (Supplementary Data 5), differential expression of DMPassociated genes was identified using a cutoff of ${ }^{*} p<0.05$ comparing MGUS/SMM/MM to healthy controls for both DNA methylation and gene expression (Fig. 2A and Supplementary Data 6). Gene ontology (GO) analysis revealed that the genes displaying both differential methylation and expression were enriched in functional categories important in cell fate commitment and bone phenotype (Fig. 2B). The most enriched functional category corresponded to genes from the Homeobox family. Within the Homeobox family, we found the subset of Hox genes that encodes a large family of highly conserved TFs responsible for driving the correct differentiation of $\mathrm{MSCs}^{33}$, namely genes belonging to the HOXA-to-D clusters. Furthermore, we observed a significant enrichment in genes reported to be downregulated in MM-MSCs (Fig. 2B) $)^{13}$. Integration of methylation and gene expression data corresponding to the Homeobox and bone formation-related genes revealed that both DNA hyper- and hypomethylation events were associated with both downregulation and upregulation of gene expression in different genomic locations (Fig. 2C). Specifically, hypermethylated genes that showed a reduced expression in patient MSCs include positive regulators of $\mathrm{OB}$ differentiation such as RUNX2 or $N_{R P} 2^{34}$ (Fig. 2C and Supplementary Table 1) In contrast, negative regulators of osteogenesis such as SFRP2 $2^{35}$ or NFATC2 ${ }^{36}$ were hypomethylated and consequently upregulated in patient MSCs (Fig. 2C). In all, these factors could potentially contribute to impaired osteoblastogenesis associated with bone disease in MM and this is summarized in Supplementary Table 1.
\end{abstract}

Upon a closer inspection of several Homeobox-associated genomic regions, we observed a negative correlation between DNA methylation of promoters and gene expression. Specifically, the HOXA gene cluster showed aberrant hypomethylation at the HOXA4 promoter, and its gene expression was upregulated at different disease stages. Conversely, gene promoters of $H O X-A 6$, $-A 7,-A 9,-A 10$, and $-A 11$ displayed hypermethylation and these genes were downregulated in MGUS/SMM/MM (Fig. 2D and Supplementary Fig. 2A). A similar pattern of an inverse association between methylation and expression was observed in the HOXB and HOXC gene cluster, where HOXB5, - C5, and -C8 were aberrantly hypomethylated and upregulated, whereas $\mathrm{HOXC9},-\mathrm{C} 10$, and -C11 were hypermethylated and downregulated in patients (Fig. 2D and Supplementary Fig. 2A). Other Homeobox genes such as TBX5, PITX1, or EMX2 were also reported as regulators of bone formation ${ }^{37-39}$ and showed an association between DNA methylation at gene promoter and gene expression (Fig. 2D and Supplementary Fig. 2A, B).

We then validated the aforementioned DNA methylation and gene expression changes in an independent cohort of BM-derived MSCs from different MM disease stages by pyrosequencing and real-time quantitative PCR. Among the differentially methylated genes of the Homeobox family, we selected HOXA2, -A4, -A6, $-A 9,-A 10,-B 7,-C 9,-C 10$, and PITX1 on the basis of their reported role in MSC pluripotency (Fig. 2E, F and Supplementary Fig. $2 \mathrm{C}, \mathrm{D})^{40}$. Furthermore, we validated differentially methylated genes with osteogenic roles in the myeloma context, including RUNX2 and IBSP (Fig. 2E, F and Supplementary Fig. 2C, D). In most cases, we observed that DNA methylation negatively correlated with gene expression.

Healthy MSCs change their DNA methylation profile to one partially resembling that of MSCs from MM patients upon interaction with MM plasma cells. To address the potential contribution of MM cells in mediating aberrant DNA methylation changes in MSCs, we evaluated whether the epigenetic changes observed in MM-MSCs could be mimicked in vitro by direct contact of healthy MSCs with MM cells. Thus, we cocultured BM-derived MSCs from healthy donors with the human MM cell line MM.1S for 2 weeks Subsequently, MSCs were sorted by $\mathrm{CD} 13^{+}$expression and subjected to DNA methylation analysis (Fig. 3A).

Under these conditions, MM.1S cells were able to induce the expression of genes known to be upregulated in MM-MSCs (IL1B, IL6, and HGF) in HD-MSCs compared with monocultured HD-MSCs (Fig. 3B). Additionally, we validated the inhibitory effect of MM cells in MSC-to-OB differentiation and observed a decrease in both ALP activity and OB mineralization in OBs differentiated in the presence of conditioned media from the MM.1S cell line as compared to OBs differentiated alone (Fig. 3C).

We then investigated the DNA methylation profiling of MSCs from healthy donors generated upon interaction with MM cells. We observed that $142 \mathrm{CpGs}$ that change their methylation levels upon co-culture with MM.1S cells were shared with aberrant DNA profiles found in MSCs isolated from MGUS/SMM/MM patients (Fig. 3D and Supplementary Data 7). Although this accounted for a small percentage of DMPs identified in MM patient MSCs, GO analyses revealed enrichment in Homeobox genes and categories related to bone formation, similar to that observed in primary patient MSCs (Fig. 3E). Specifically, we found that healthy MSCs exposed to MM cells underwent gains (HOXA9, ACVR2A, EBF2) and losses (HOXA2, HOXA3, HOXC5) of DNA methylation in the direction of those observed for MSCs from myeloma patients (Fig. 3F). 

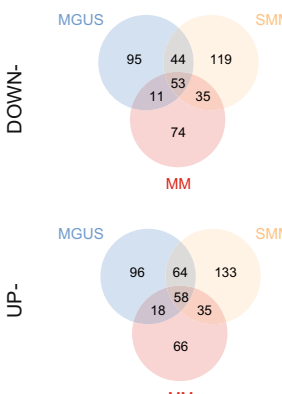

B

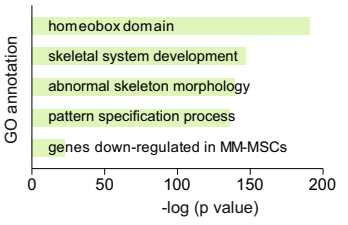

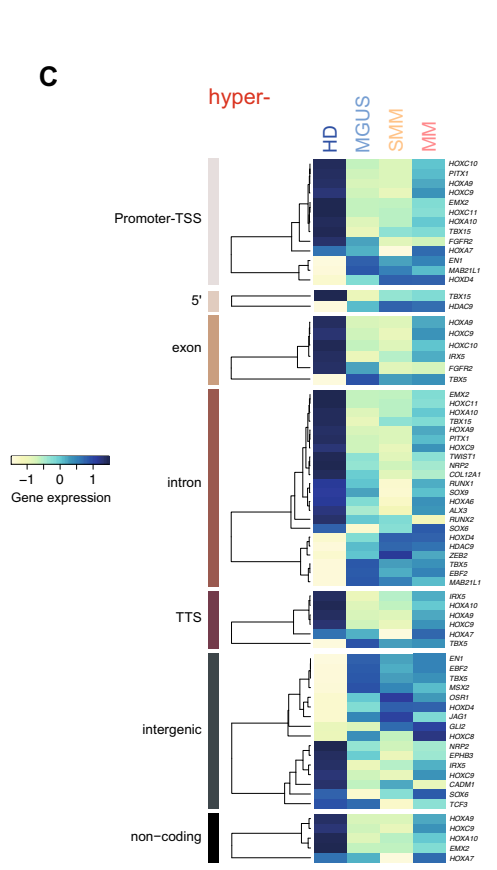

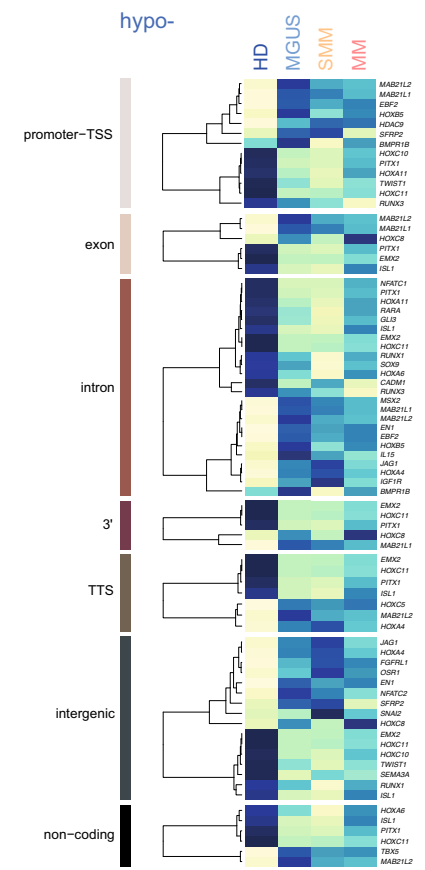

D

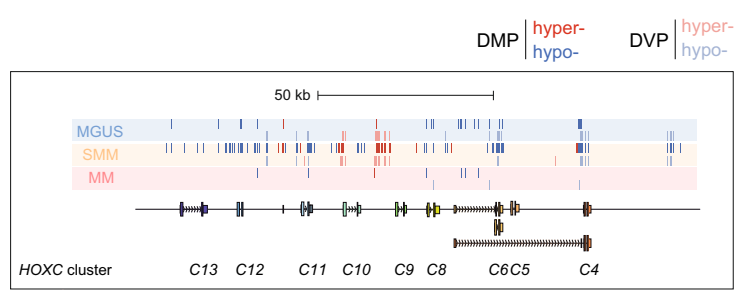

$\begin{array}{lllllllllll}\text { HOXA cluster } & A 1 & A 2 & \text { A3 } & \text { A4 } & \text { A5A6 } & \text { A7 } & \text { A9 } & \text { A10 } & \text { A11 } & \text { A13 }\end{array}$
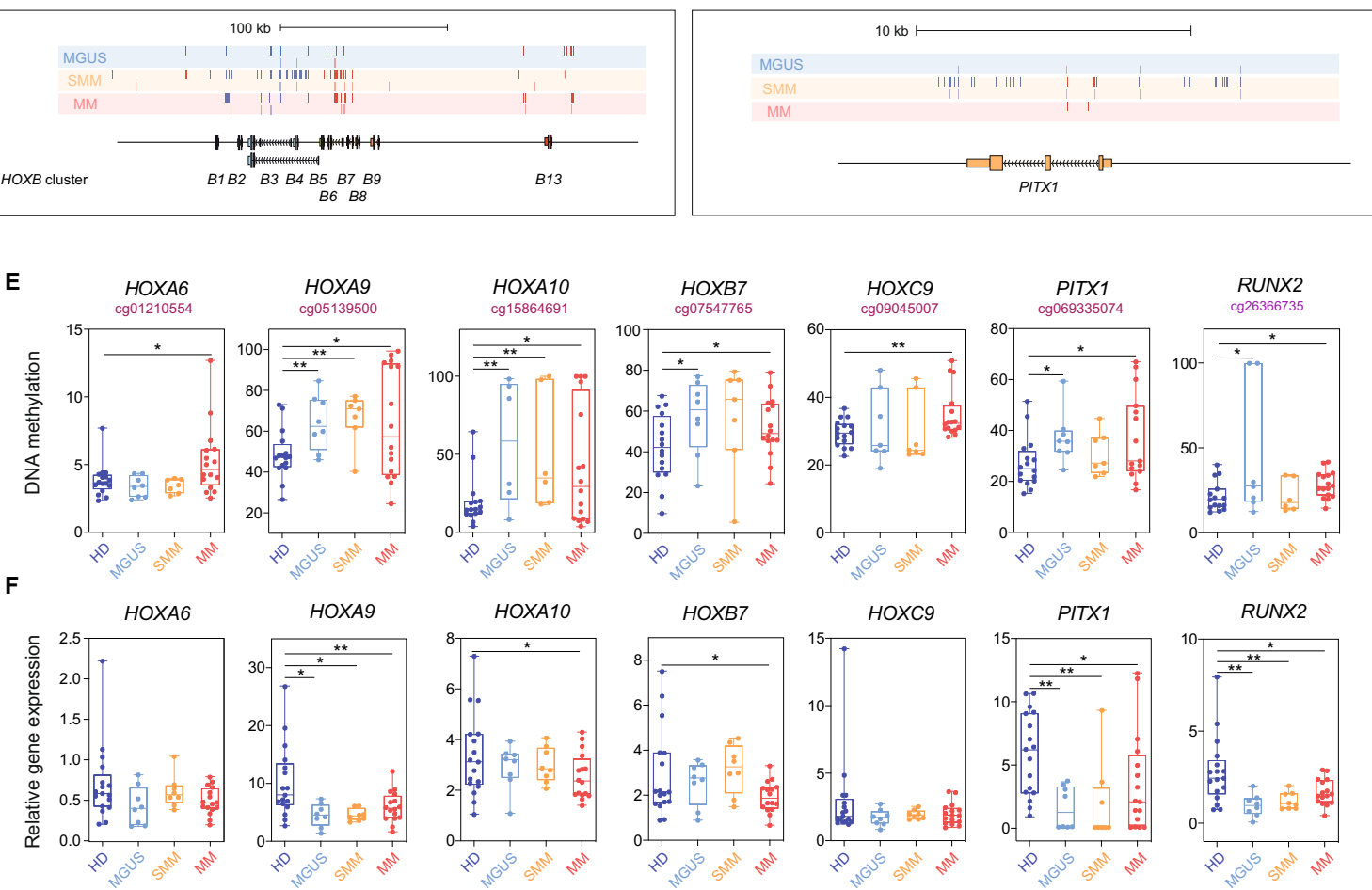

Moreover, we validated the DNA methylation and gene expression changes in healthy MSCs driven by co-culture with MM.1S with another MM cell line, RPMI-8226. Here, we observed a similar effect of co-culture with RPMI-8226, in which there was a clear inhibition of Homeobox and osteogenic gene expression coupled with hypermethylation of these loci (Fig. 3G, H).

Altogether, these results support the notion that MM cells not only are capable of inducing changes in the global methylome of MSCs but also have a significant impact at specific osteogenic loci. 


\begin{abstract}
Fig. 2 DNA methylation changes are associated with differential gene expression of Homeobox genes in MSCs from MGUS, SMM and MM patients. A Venn diagrams showing differentially methylated or downregulated (upper) and upregulated (lower panel) genes when comparing MGUS (blue), SMM (orange), and MM (red) samples with healthy individuals. B Gene ontology (GO) enrichment analysis of CpG sites undergoing DNA methylation and gene expression changes in MSCs of patients compared to controls using the GREAT online tool. A binomial test was performed to calculate statistical significance. C Heatmaps showing gene expression of Homeobox and other OB-related genes associated with differentially hyper- (left) or hypomethylated (right) CpG sites. Heatmaps are grouped according to the genomic location (promoter, TSS, 5', exon, intron, intergenic, 3' or non-coding region) of analyzed $\mathrm{CpG}$ sites. Color scale ranging from light yellow to dark blue represents low to high expression levels. D Scheme depicting differentially methylated and variable CpG sites located in the Homeobox genes (HOX-A, -B, and -C clusters) and PITX1. Dark blue lines indicate hypomethylated DMPs, light blue lines indicate hypomethylated DVPs, dark red lines indicate hypermethylated DMPs and light red lines indicate hypermethylated DVPs associated with MGUS, SMM, and MM condition. E DNA methylation analysis by pyrosequencing of selected CpGs located at the promoter regions and $\mathbf{F}$ gene expression of HOXA6, -A9, -A10, -B7, -C9, PITX1, and RUNX2 in MSCs from healthy controls (dark blue; $n=17$ ), MGUS (light blue; $n=8$ ), SMM (orange; $n$ $=8)$, and MM $(n=16)$ patients. Gene expression was normalized against RPL38. Box plots represent median \pm IQR and whiskers represent maximum and

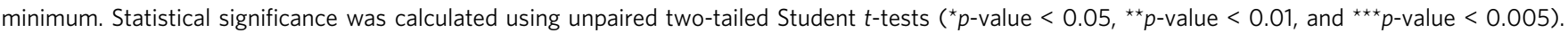

Dual targeting of DNMTs and G9a restores Homeobox gene expression in vitro and promotes osteogenic differentiation of mesenchymal precursors. Gene expression analysis of DNMTs in MSCs from HD and MM patients co-cultured with MM cells obtained from a previous study ${ }^{41}$ showed an aberrant upregulation of the DNA methyltransferase DNMT1 (Fig. 4A). DNMT1 interacts with the methyltransferase G9a to coordinate DNA and H3K9 methylation during cell replication ${ }^{42}$ promoting transcriptional silencing of target genes. Moreover, G9a can suppress transcription by inducing DNA methylation in addition to its activity as a chromatin remodeler ${ }^{43}$. In this regard, we hypothesized that the dual inhibition of DNMT1 and G9a could reactivate hypermethylated and silenced genes of MSCs from MM patients preserving their osteogenic potential and therefore preventing myeloma-associated bone loss. Thus, we utilized a dual inhibitor of DNMTs and G9a, termed CM-272, which has been previously described to have a potent therapeutic response, both in vitro and in vivo, in other neoplasias ${ }^{44-47}$.

We first checked the effect of CM-272 on the cell viability of mesenchymal progenitors and we selected a dose $(50 \mathrm{nM})$ with no significant toxicity in order to perform further experiments (Fig. 4B). CM-272 treatment was able to restore the expression of Homeobox genes (HOX-A6, -A9, -A10, -C9, PITX1, and RUNX2) that were epigenetically repressed in MSCs from MM patients (Fig. 4C). Mechanistically, we observed a loss of DNA methylation in the promoter region of the majority of the aforementioned genes after CM-272 treatment in MM-MSCs (Fig. 4D). We then checked the levels of the inactive chromatin mark H3K9me2, a hallmark of methyltransferase G9a activity, at these gene promoters upon CM-272 treatment. The chIP-qPCR analysis showed a decrease in $\mathrm{H} 3 \mathrm{~K} 9 \mathrm{me} 2$ levels at the promoter regions of Homeobox genes after CM-272 treatment (Fig. 4E). Taken together, our results suggest that CM-272 acts in vitro by inhibition of both DNMT and G9a methyltransferase activity.

Next, we addressed whether targeting DNMT and G9a may have a role in regulating osteogenic differentiation. For this purpose, we cultured MSCs from myeloma patients in osteogenic media to obtain differentiated OBs in the presence or absence of CM-272. As observed in Fig. 4F, G, CM-272 was able to increase ALP activity in early-stage OBs. Furthermore, CM-272 treatment was able to upregulate the relative expression of several late bone formation markers (namely, bone siaploprotein, osteopontin, and osteocalcin) in MSCs from myeloma patients (Fig. 4H).

Previous research has described that MM cells exert their effect on MSCs through both direct cell-cell contact and soluble factor mechanisms ${ }^{11,48}$. Our main data show that direct myeloma-MSC co-culture conditions are able to induce changes in the MSC methylome; however, it would be of particular interest to investigate whether MM cells may also mediate the same changes only through soluble factor mechanisms. For this purpose, utilizing a transwell system avoiding contact between both cell types, we observed that soluble factor secreted by MM.1S and RPMI-8226 cell lines were sufficient to change the expression of several OB-relevant genes in healthy MSCs, including RUNX2, SPP1, IBSP, and HOXB7 (Fig. 5A), concordantly to direct coculture. Furthermore, treatment with the dual inhibitor CM-272 was able to partially reverse those changes in gene expression mediated by soluble factors secreted by MM cells (Fig. 5A). Changes in gene expression were accompanied by inverse changes in DNA methylation in some of the genes (IBSP, HOXB7) (Fig. 5B), which were also observed to at least be partially reversed by CM-272. However, for some other genes such as RUNX2 and SPP1, transwell co-culture with MM cells induced minimal effect on DNA methylation, suggesting that direct cell-cell contact may be required. Moreover, CM-272 was able to partially reverse the MM cell lines-mediated inhibitory effect on $\mathrm{OB}$ mineralization (Fig. 5C). Altogether, these results suggest that MM cells at least partially exert its effects on MSCs through secretory mechanisms, and treatment with CM-272 was able to reserve these effects through the inhibition of DNA methylation.

CM-272 not only controls tumor burden but also prevents the myeloma-associated bone loss. To test the effect of CM-272 in the context of MBD, we used an established murine model of bone marrow-disseminated myeloma. After equivalent engraftment of myeloma cells (RPMI-8226-luc) was verified by bioluminescence measurement, mice were treated for 4 weeks with CM-272 as described in Methods. Compared with the vehicle control group, CM-272 controlled tumor progression as measured by bioluminescence (Fig. 6A) or by serum levels of hIg $\lambda$ secreted by RPMI-8226 cells (Fig. 6B). Representative microCT images at the metaphyses of distal femurs showed a tumorassociated bone loss in vehicle-treated mice, in contrast with trabecular structures observed in CM-272-treated animals (Fig. 6C). In the vehicle control group, 3D reconstruction images of distal femurs revealed a marked bone loss evidenced by a thin trabecular network (in red) but also by loss of cortical bone (in gray) in vehicle-treated mice (Fig. 6D). By contrast, CM-272treated mice presented a gain in both trabecular and cortical bone (Fig. 6D). This was also reflected by bone morphometric parameters that resulted in increased trabecular bone volume, occupancy, and connectivity and reduced trabecular separation in CM-272-treated animals, as compared with vehicle control (Fig. 6E). Finally, these findings correlated with a significant increase in serum levels of the bone formation marker P1NP analyzed after CM-272 treatment compared to untreated control (Fig. 6F). In summary, these data demonstrate that CM-272 exerts in vivo anti-myeloma activity along with bone-anabolic effects in human MM-bearing mice. 
A
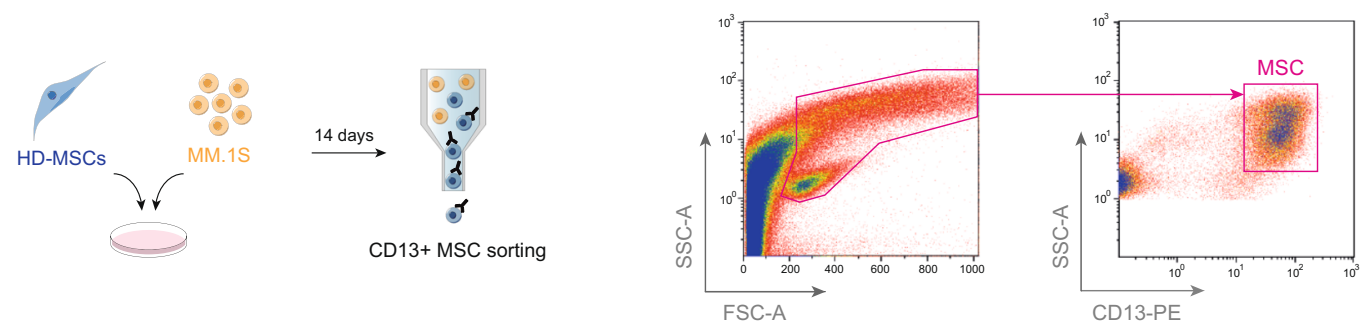

B

C
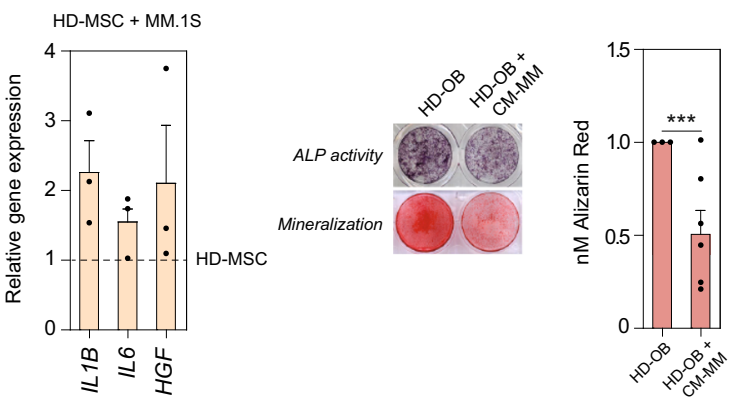

D

hyper-

E

$\mathbf{F}$

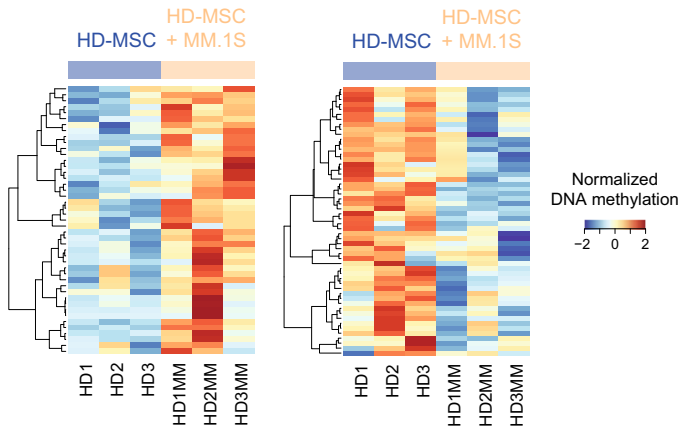

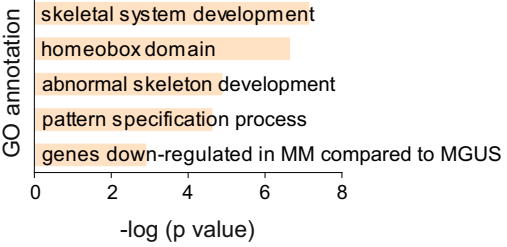

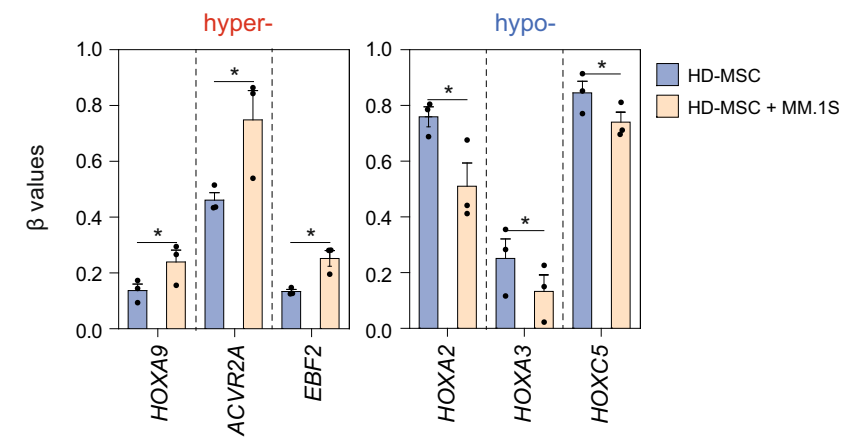

G
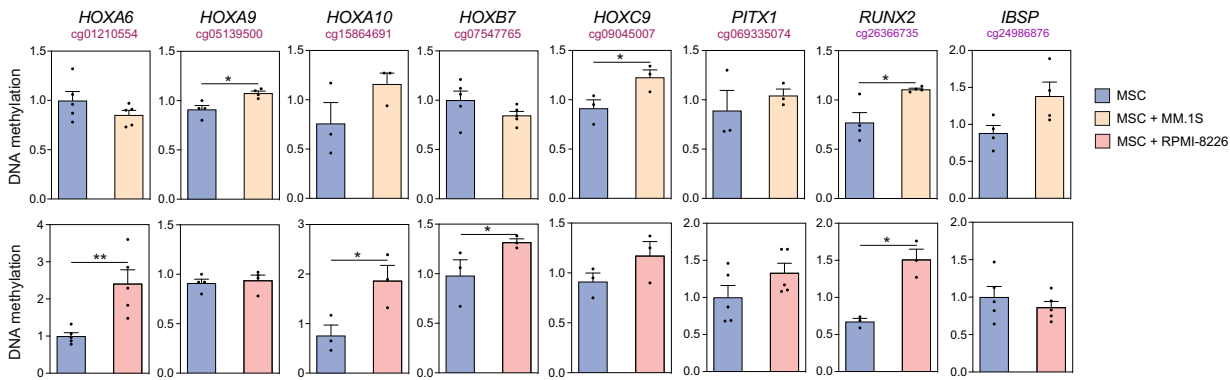

H

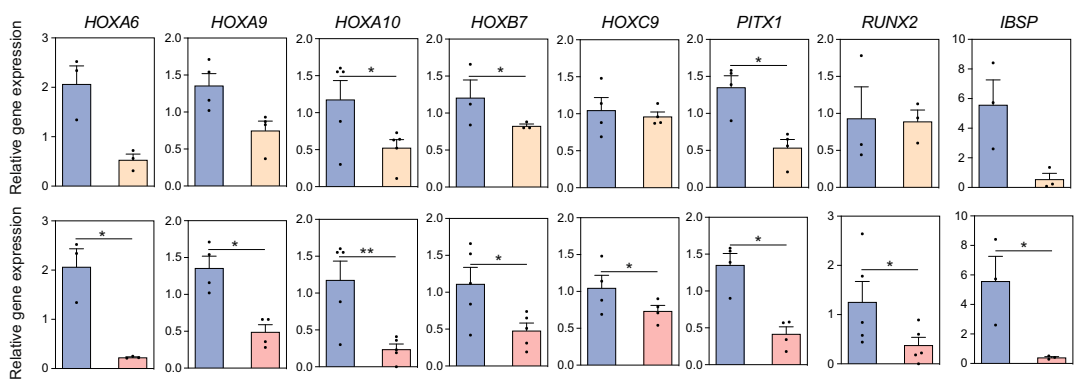

To further examine the in vivo effect of CM-272 on DNA methylation of myeloma-associated MSCs, we performed reduced representation bisulfite sequencing (RRBS) analysis of MSCs isolated from vehicle- and CM-272-treated myeloma-bearing mice using healthy mice as controls. First, we observed significant alterations in the DNA methylome of MSCs from myelomabearing mice compared to healthy mice (Supplementary Data 8).
Myeloma-bearing mice that were treated with CM-272 displayed a partial reversion of aberrant hypermethylation of MSCs caused by the presence of myeloma cells (Fig. 6G). These DNA methylation changes occurred at genomic loci enriched for genes involved in cell commitment and differentiation, such as Homeobox genes (Fig. 6H). Specifically, we were able to identify CpGs that experienced a gain in DNA methylation in vehicle- 
Fig. 3 MSCs from healthy donors recapitulate DNA methylation changes observed in MSCs from MM patients upon interacting with MM plasma cells. A Scheme depicting workflow (left panel) and sorting strategy (right panel) for selecting CD13 + MSCs after 14 days of co-culture with the MM.1S cell line. B Gene expression analysis of $I L 1 B, I L 6$, and HGF, normalized against RPL38, of the hMSC-TERT cell line (HD-MSC) co-cultured with the MM.1S cell line for 14 days. Expression levels were normalized against MSCs in monoculture. Data are represented as the mean \pm SEM from three independent experiments. C ALP activity and matrix mineralization were assessed in differentiated osteoblasts from HD-MSCs in the presence (HD-OB + CM-MM) or absence (HD$\mathrm{OB}$ ) of conditioned media from the MM.1S cell line (CM-MM). Representative images of each experimental condition are shown. Barplot represents the mean \pm SEM from six independent experiments, and a paired two-tailed $t$-test was performed to evaluate statistical significance $\left({ }^{\star \star \star} p<0.005\right)$. D Heatmap showing differentially methylated $\mathrm{CpG}$ sites (eBayes-moderated paired $t$-test ${ }^{\star} p<0.05$ ) in sorted HD-MSCs (three independent donors) in monoculture (HD1-3) or co-cultured with the MM.1S cell line (HD1-3MM) for 14 days that overlaps with previously identified DMPs. The color scale from blue to red represents low to high methylation levels. E GO enrichment analysis of DMPs in HD-MSCs co-cultured with the MM.1S cell line overlapping with myelomaassociated DMPs analyzed using the GREAT online tool. $p$-values were calculated using a binomial test. F Bar plots showing $\beta$-values obtained from the DNA methylation array presented in $\mathbf{D}$, representing mean and \pm SEM of three independent experiments. $\mathbf{G}$ DNA methylation and $\mathbf{H}$ gene expression levels of DMPs validated in HD-MSCs monoculture (blue) or co-cultured with MM.1S (orange) or RPMI-8226 (red) cell lines as indicated. Gene expression data were normalized against RPL38 and all data were normalized against HD-MSC monoculture. Statistical significance was calculated using paired one-tailed Student $t$-tests and bar plots represent mean \pm SEM of 3-5 independent experiments ( ${ }^{\star} p$-value $<0.05,{ }^{\star \star} p$-value $<0.01$, and ${ }^{\star \star \star} p$-value $<0.005$ ).

treated MSCs compared to healthy controls at the same genomic loci previously identified in human MM-MSCs, including HOXA7, $-B 4,-B 7,-B 9$, and $-D 10$ (Fig. 6I and Supplementary Data 9). Importantly, CM-272 treatment was able to restore the DNA methylation levels at these loci to resemble that of healthy mice, which was concomitant with the reduced tumor burden as well as bone loss recovery observed in these mice (Fig. 6I).

\section{Discussion}

The pathogenic transition from premalignant stages to active $\mathrm{MM}$ is complex and not well understood. One example of this complexity is that although all MM cases emerge from the preexisting asymptomatic MGUS/SMM stage, not all MGUS progress into MM and may exist as a stable and independent disease. Nevertheless, despite being an asymptomatic stage, transformed plasma cells in MGUS present cytogenetic alterations similar to that of myeloma plasma cells, as well as significant abnormalities in bone remodeling ${ }^{49,50}$. This indicates that both genetic and microenvironmental alterations exist from the early stages of the disease. In our study, we show that epigenetic alterations in MSCs already occur in the early asymptomatic stages of both MGUS and SMM, and although many alterations are shared between all stages, the majority of DNA methylation changes are specific to each stage. These results are in accordance with previous studies that indicate the existence of stage-specific epigenetic alterations during MM progression in malignant plasma cells ${ }^{51,52}$. This phenomenon could be explained by the expansion of subpopulations of MSCs during MM disease progression, which may favor tumor development and drug resistance, similar to what was observed to occur in MM cells ${ }^{53,54}$.

Deregulation of methylome in MM-MSCs mediates transcriptional and phenotypical alterations. Interestingly, many genes of the Homeobox family displayed both epigenetic and transcriptional dysregulation in patient MSCs, and these changes were observed in earlier stages of the disease. In this regard, members of the HOX family have been recently described to be key drivers of $\mathrm{OB}$ differentiation, in which their expression is fine-tuned by demethylation of their promoters during the osteogenic process ${ }^{55}$. Furthermore, we observed that healthy MSCs exposed to MM cells, similarly to that observed in patient MSCs, not only displayed an altered methylome but also showed impaired MSC-to-OB differentiation, as previously described ${ }^{20}$. We also observed that some of these methylome changes in MSCs occur in the absence of direct cell-cell contact with MM cells, suggesting the contribution of secretory mechanisms. Hence, our results suggest that the impairment of osteogenesis in all stages of
MM arises from early transcriptional deregulation of Homeobox genes, and altered DNA methylation may be the primary mediator in this process. Nevertheless, we cannot overlook the limitations of our in vitro studies, as other cell types of the BM microenvironment may also play important roles in perpetuating the methylome alterations observed in MSCs.

Although the biology of MBD is relatively well described, there is still a lack of pharmacological treatments to improve bone loss. Clinically approved bone-modifying agents for the treatment of MBD include bisphosphonates ${ }^{56}$, which inhibit bone resorption by suppressing OC activity, and denosumab ${ }^{57}$, a monoclonal antibody against the osteoclastogenic cytokine RANKL. However, these drugs only target the OC compartment, and bone disease persists due to the absence of bone formation. Thus, therapeutic agents targeting OBs are needed. In this study, we demonstrated a strategy for treating MBD by targeting aberrant DNA methylation in MSCs. Firstly, we showed that co-culture of healthy MSCs with MM cell lines yielded epigenetic and transcriptional changes similar to that observed for MSCs from myeloma patients, and treatment with CM-272 was able to at least partially reverse these changes. Additionally, this agent promoted the ability of MSCs to differentiate into OBs. These in vitro effects on bone were mirrored in a mouse model of disseminated MM. Of note, CM-272 treatment not only prevented bone loss by bone-anabolic effects but also showed anti-myeloma activity. This is in line with previous reports showing that DNMTs are targets for the treatment of $\mathrm{MM}^{58-60}$ and also for improving the osteogenic differentiation ability of MSCs ${ }^{61}$. Additionally, we cannot discard the possibility that the observed effects on tumor growth inhibition may be a consequence of the impairment of the cross-talk between MSCs and MM cells. Moreover, the dual targeting effects of CM-272 also inhibit the dimethylation of $\mathrm{H} 3 \mathrm{~K} 9$, which has been described to be crucial in the establishment of DNA methylation ${ }^{42,43}$. It is therefore rational to envision that the bone-anabolic effects mediated by CM-272, both in vitro and in vivo, involves the reversion of aberrant hypermethylation at Homeobox loci and other OB-related genes in the MSC population. Nevertheless, it is possible that reduced tumor burden could be partially responsible for restoring the bone-forming capacities of MM-MSCs.

In summary, our findings highlight the existence of aberrant DNA methylation patterns in the BM-derived MSC population which may impact myeloma progression and the development of MBD. Moreover, our preclinical results support the idea that therapeutic targeting of aberrant DNA methylation would result in an anti-myeloma effect and preservation of the appropriate osteogenic differentiation of MSCs to combat myeloma bone disease. 
A

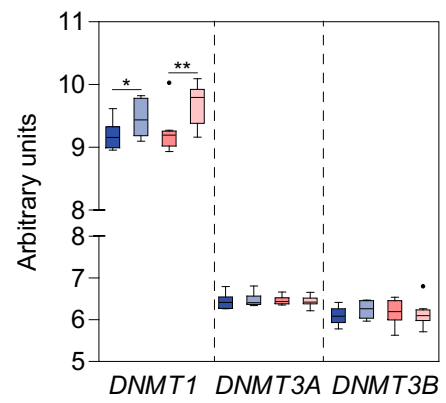

B

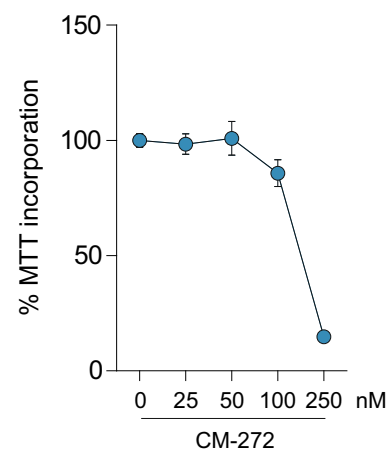

C

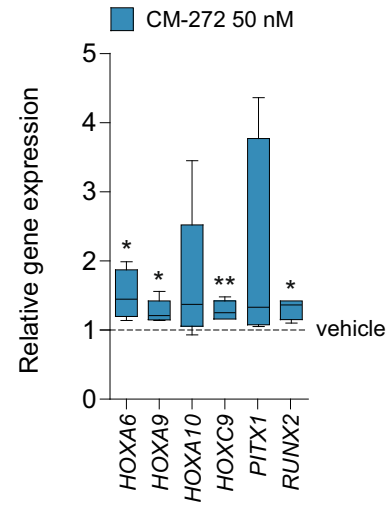

D
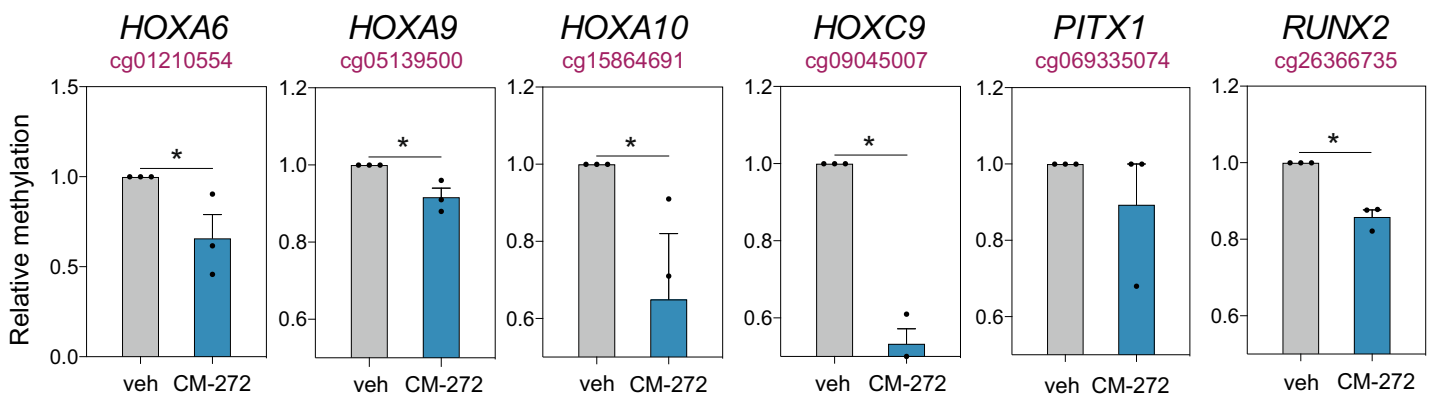

E
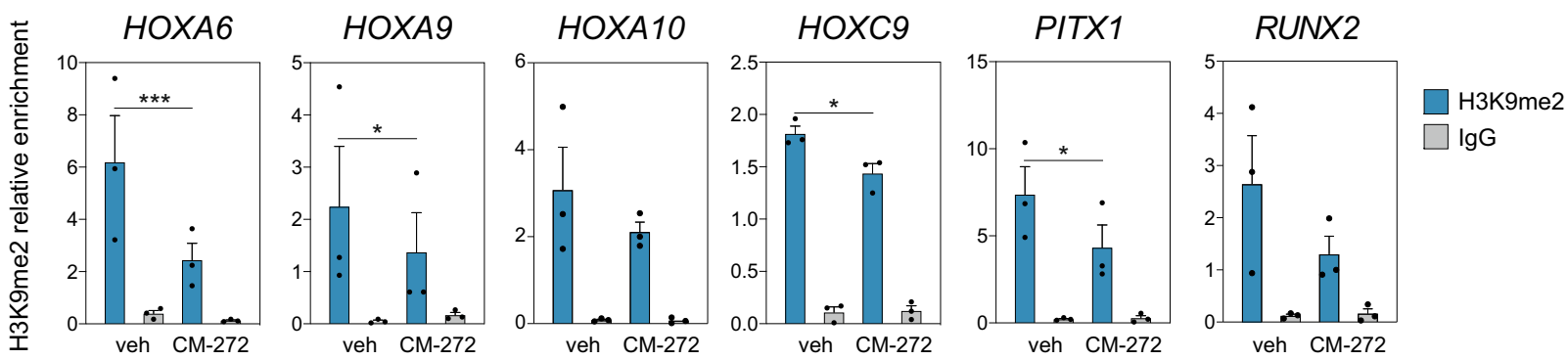

$\mathbf{F}$

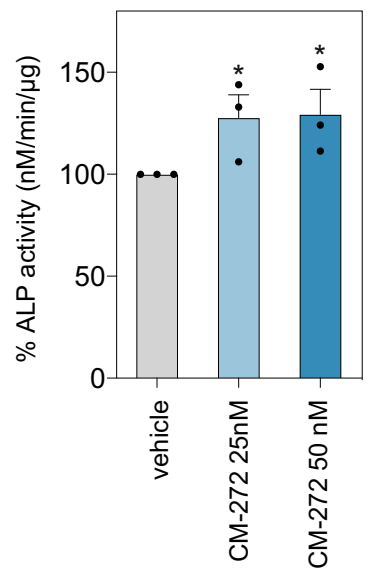

G

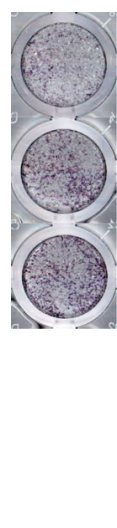

H

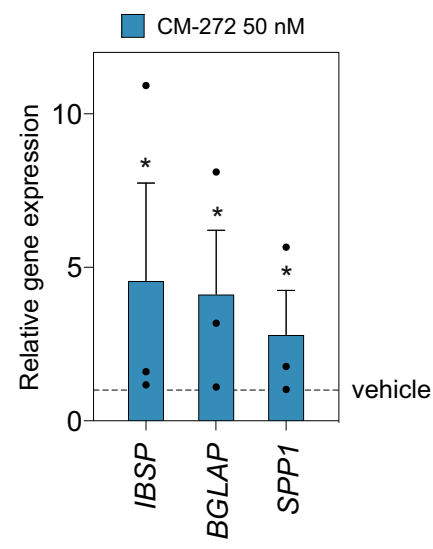

\section{Methods}

Participants. BM samples were obtained from the iliac crest of patients with newly diagnosed MGUS $(n=10)$, SMM $(n=8)$, and MM $(n=9)$, according to the International Myeloma Working Group criteria. BM samples from healthy controls $(n=8)$ were obtained from participants undergoing orthopedic surgery not related to oncology disease. Each sample was obtained after receiving the informed written consent of all participating subjects and following approval from the committees listed below for obtaining them and for the study protocol using them. The study was approved by the Cancer Research Center-IBMCC Review Board (CICIC 2015/
02156), the Clinical Ethics Committee for drug research in the Salamanca Health Area (CEIC 73/07/2015), the Clinical Research Committee of the Bellvitge University Hospital (ref. PR076/15) and the Research Ethics Committee of the University of Navarra (ref. 2017.218). Clinical characteristics of MGUS, SMM, and MM patients are listed in Supplementary Table 2.

Inhibitor. CM-272 (dual DNMTs and G9a inhibitor) was synthesized at the Center for Applied Medical Research (University of Navarra) ${ }^{44,47,62}$. 


\section{Fig. 4 CM-272 treatment reactivates Homeobox gene expression and promotes the osteogenic differentiation of MSCs from MM patients. A} Expression of DNA methyltransferases 1, 3A, and B (DNMT1, -3A, and -3B) in HD and MM-MSCs co-cultured for $24 \mathrm{~h}$ with the MM.1S cell line comparing with monoculture as assessed by the GeneChip Human Gene 1.0 ST Array. Box plots present mean \pm SEM for 8 healthy donors and 9 MM patients. $p$-values were calculated by paired two-tailed Student $t$-test $\left({ }^{\star} p<0.05,{ }^{\star \star} p<0.01\right)$. B MSCs from MM patients were treated with the indicated doses of CM-272 for $72 \mathrm{~h}$ and subjected to MTT assay for viability. Mean and SEM are indicated on the line chart from 3 independent experiments. C Real-time RTPCR was performed to determine the expression of hypermethylated Homeobox genes (HOX-A6, -A9, -A10, -C9, PITX1, RUNX2) in MM-MSCs treated with vehicle or $50 \mathrm{nM}$ of $\mathrm{CM}-272$ for 7 days. Box plots represent median $\pm \mid \mathrm{QR}$, with whiskers representing minimum and maximum, of 9 independent experiments. A paired two-tailed Student's $t$-test was performed to calculate statistical significance $\left({ }^{\star} p<0.05,{ }^{\star \star} p<0.01\right)$. D DNA methylation analysis by pyrosequencing of selected CpGs located at the promoter regions of Homeobox genes in MM-MSCs treated with vehicle (gray) or CM-272 (blue) for 7 days. Bar plots present mean \pm SEM for 3 independent experiments and paired two-tailed Student's $t$-tests were performed $\left({ }^{*} p<0.05\right)$. E ChIP assays showing the H3K9me2 (blue) enrichment at the promoter regions of Homeobox genes in MM-MSCs treated with vehicle or CM-272 for 7 days. IgG (gray) was used as a negative control. Data are shown as a relative enrichment of the bound fraction with respect to the input DNA. Bar plots present mean \pm SEM for 3 independent experiments and paired two-tailed Student's $t$-tests were performed $\left({ }^{\star} p<0.05,{ }^{\star \star} p<0.01\right.$, and $\left.{ }^{\star \star \star} p<0.005\right)$. ALP activity was assessed in MM-MSCs $(n=3)$ cultured in osteogenic media in the presence of $25 \mathrm{nM}$ (light blue) and $50 \mathrm{nM}$ (dark blue) of CM-272, compared to vehicle (gray), by $\mathbf{F}$ p-NPP hydrolysis and G NBT-BCIP. H Expression of osteoblastogenesis markers IBSP (bone sialoprotein), BGLAP (osteocalcin) and SPP1 (osteopontin) was checked by qRT-PCR in MM-MSCs cultured in osteogenic media in the absence (vehicle) or presence of CM-272. For $\mathbf{F}$ and $\mathbf{H}$ data are shown as mean values \pm SEM from three different experiments. Statistically significant tests (paired two-tailed Student's $t$-tests) are represented as ${ }^{\star} p<0.05,{ }^{\star \star} p<0.01$, and ${ }^{\star \star \star} p<0.005$ between vehicle and $C M-272$ condition.

Cell lines. The human multiple myeloma cell line MM.1S was provided by Dr. Steven Rosen (Northwestern University, Chicago, IL), whereas RPMI-8226 cells were purchased from the American Type Culture Collection. The human mesenchymal stem cell (MSC) line immortalized by expression of the telomerase reverse transcriptase gene (hMSC-TERT) was a generous gift from Dr. D Campana (Department of Pediatrics, Yong Loo Lin School of Medicine, National University of Singapore, Singapore). Both cell lines were cultured in RPMI 1640 medium supplemented with $10 \%$ heat-inactivated fetal bovine serum (FBS), $100 \mathrm{U} / \mathrm{ml}$ penicillin and $100 \mathrm{mg} / \mathrm{ml}$ streptomycin, and $1 \%$ L-glutamine. All the cell culture media and reagents were purchased from Invitrogen (Paisley, UK). All cell types were cultured at $37^{\circ} \mathrm{C}$ in a humidified atmosphere in the presence of $5 \%$ $\mathrm{CO}_{2}-95 \%$ air.

Bone marrow-derived MSC isolation and culture. MSCs were isolated and characterized as described by Garayoa et al. ${ }^{17}$. Briefly, bone marrow aspirates were obtained from the iliac crest and subjected to centrifugation on Ficoll-Paque (GE Healthcare, Uppsala, Sweden) to obtain mononuclear cells (BMMCs). BMMCs were plated and plastic-adherent cells were expanded until passage 3 (P3) in lowglucose DMEM supplemented with $10 \%$ heat-inactivated fetal bovine serum, 100 $\mathrm{U} / \mathrm{ml}$ penicillin, $100 \mathrm{mg} / \mathrm{ml}$ streptomycin, and $1 \% \mathrm{~L}$-glutamine. Selected MSCs from both MM patients $(n=4)$ and healthy donors $(n=4)$ at P3 were tested to meet minimal criteria as defined by the International Society for Cellular Therapy for multipotent mesenchymal stromal cells ${ }^{63}$. Specifically, MSCs were evaluated by FACS for positive expression of CD73, CD90, CD105, CD44, and CD166 and negative staining for HLA-DR and hematopoietic markers (CD19, CD34, and CD45) (Supplementary Fig. 3A). In addition, the capability to differentiate into osteoblast, adipocyte, and chondrocyte was assessed (Supplementary Fig. 3B-D), Analyses and experiments were performed with MSCs at P3, with a maximum of 3 weeks at each passage.

DNA and RNA isolation and quantification. Genomic DNA was isolated by the proteinase K method or using the Maxwell ${ }^{\oplus}$ RSC Cell DNA Purification Kit (Promega) for samples containing low cell number. RNA was isolated using Maxwell ${ }^{\oplus}$ RSC simplyRNA Cells Kit (Promega) according to the manufacturer's instructions. DNA and RNA were quantified using Qubit ${ }^{\circledR}$ DNA Assay Kit (Invitrogen) or NanoDrop ND-1000, respectively.

DNA methylation and gene expression profiling using arrays. DNA samples were bisulfite-converted using an EZ DNA methylation kit (Zymo Research, Orange, CA) and hybridized onto an Infinium MethylationEPIC BeadChip array (Illumina, Inc.). The array platform allows the assessment of DNA methylation status at $>850,000 \mathrm{CpG}$ sites at single-nucleotide resolution and covers $99 \%$ of RefSeq genes and $95 \%$ of $\mathrm{CpG}$ islands with an average number of six probes per island.

RNA samples were obtained from healthy donors $(n=8)$, MGUS $(n=10)$, SMM $(n=10)$, and MM patients $(n=24)$ at diagnosis and $100 \mathrm{ng}$ of excellent quality RNA (RIN >9) was hybridized onto a GeneChip Human Gene 1.0 ST (Affymetrix).

Quality control, data normalization, and detection of differentially methylated and variable CpGs. Methylation array data were processed in the statistical language R v4.0 in RStudio 1.3 (https://rstudio.com) using methods from the Bioconductor libraries minfi (v1.36.0), lumi (v2.42.0), and limma (v3.46.0) ${ }^{64-66}$.
Probes were annotated using IlluminaHumanMethylationEPICmanifest v0.3.067. Data quality was assessed using the standard pipeline from the minfi package. The data were quantile-normalized and chromosomes $\mathrm{X}$ and $\mathrm{Y}$ were removed to avoid technical and biological bias. Furthermore, we discarded the DNA methylation changes associated with the long-term culture of BM-MSCs based on the previous studies ${ }^{68} . M$ values ( $\log _{2}$-transformed $\beta$-values) were utilized to obtain a $p$-value between sample groups by an eBayes-moderated paired $t$-test using the limma package, in which age and sex were added in the interaction matrix. For the analysis of MSCs isolated from MM patients and healthy controls, we considered a probe to be differentially methylated when the difference between the mean of $\beta$ at disease versus control was over $10 \%(\Delta \beta \geq 0.1)$ and the statistical test was significant $(* * p<0.01)$. In addition, we used the iEVORA algorithm ${ }^{69}$, provided by matrixTests v0.1.9 (https://CRAN.R-project.org/package=matrixTests), to designate a probe as differentially variable. This algorithm detects the homogeneity of variances using the Bartlett's test (FDR $<0.05)$ and then selects those probes whose $t$-test is significant $\left({ }^{*} p<0.05\right)$ in order to regularize the variability test which is overly sensitive to single outliers.

To evaluate the contribution of various covariates, including age and sex, we performed either a Pearson correlation or Wilcoxon signed-rank test depending on whether the covariate of interest was continuous or categorical. This is represented in Supplementary Fig. 4 , in which a covariate with a $p$-value $<0.05$ was considered to significantly contribute to DNA methylation.

For the direct co-culture of healthy MSCs with MM cell line, samples were normalized utilizing Noob and Quantile normalizations provided by minfi. The paired analysis was performed and a probe was considered differentially methylated if $\Delta \beta$ was more than $10 \%$ and $p$-value was $<0.01$

Gene ontology, motif, and chromatin state analysis. Functional annotation enrichment analysis was performed using GREAT tool v4.0.4 (http://great.stanford. edu/public/html $)^{70}$ by mapping differentially methylated $\mathrm{CpG}$ site to the single nearest gene. CpGs annotated in the EPIC $850 \mathrm{~K}$ array were used as background. GO categories with $p$-value of $<0.01$ were considered significantly enriched.

For TF binding motif analysis, HOMER motif discovery software v4.5 was used $^{27}$, where a $500 \mathrm{bp}$-window upstream and downstream of the differentially methylated $\mathrm{CpG}$ sites was applied. CpGs annotated in the EPIC array were used as background.

To analyze chromatin states associated with DMPs, ChromHMM ${ }^{26}$ data sets from healthy donor MSCs were downloaded from the UCSC Genome Browser (https://genome.ucsc.edu/). Overlap was performed in R using the GenomicRanges package v1.42.0 ${ }^{71}$, where CpGs annotated in the EPIC array were used as background.

Gene expression array normalization and analysis. Data processing and normalization were carried out using the R statistical language. Background correction was performed using Robust Microarray Analysis (RMA) normalization provided by oligo package v1.54.1 ${ }^{72}$ and probes were annotated utilizing the hugene10sttranscriptcluster.db R package v8.7. $0^{73}$. Average expression was calculated for probes mapping to the same gene. For comparisons between groups, eBayesmoderated $t$-test provided by the limma $\mathrm{R}$ package ${ }^{65}$ was applied, where a $p$-value $<0.05$ was considered statistically significant. DMPs were mapped to the nearest gene utilizing the GREAT online tool, and overlap with differentially expressed genes were performed by overlapping gene names. 
A
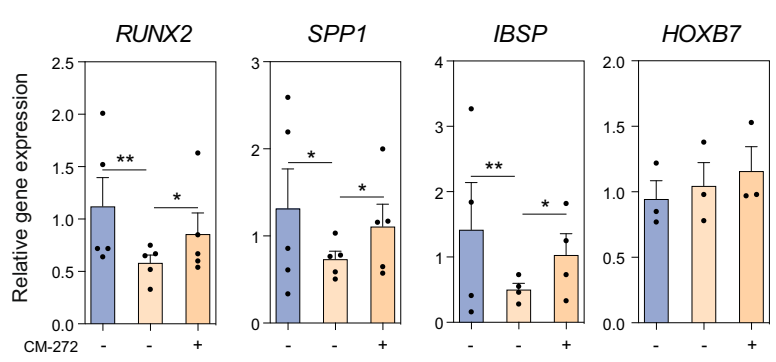

MSC

\section{$M S C+M M .1 S$}

MSC + RPMI-8226

C
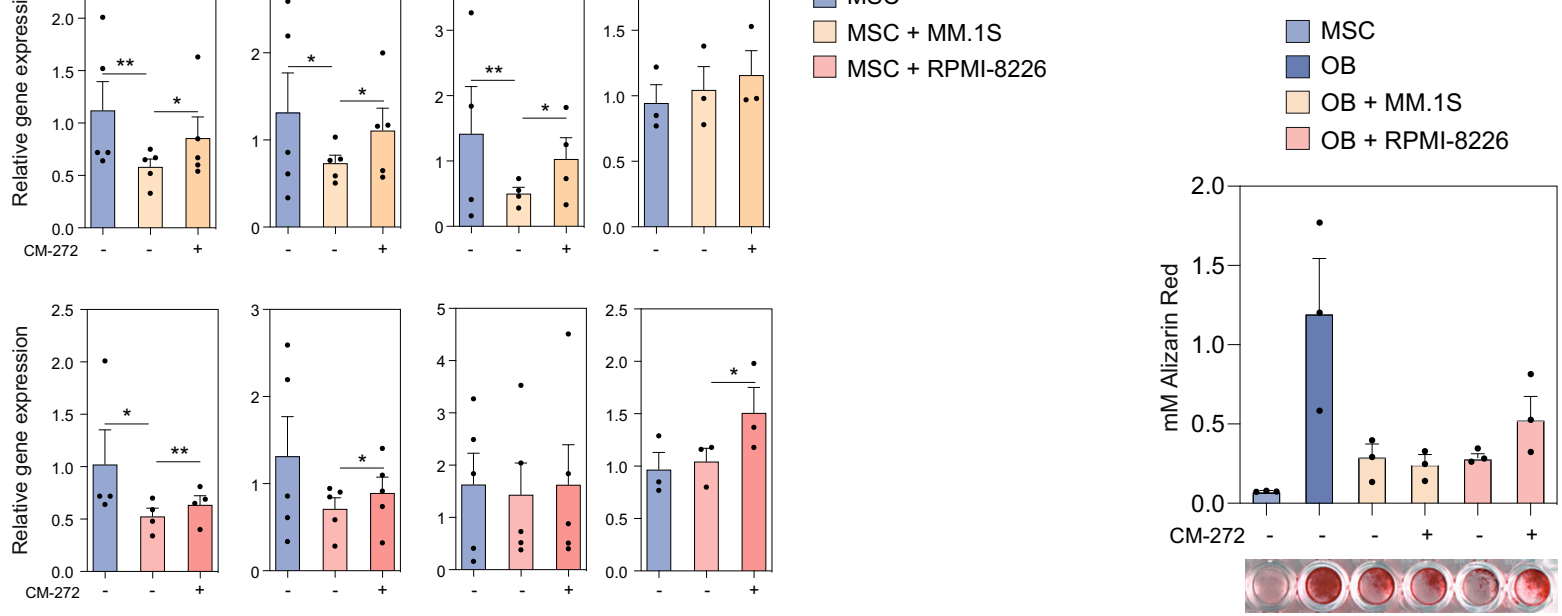

B
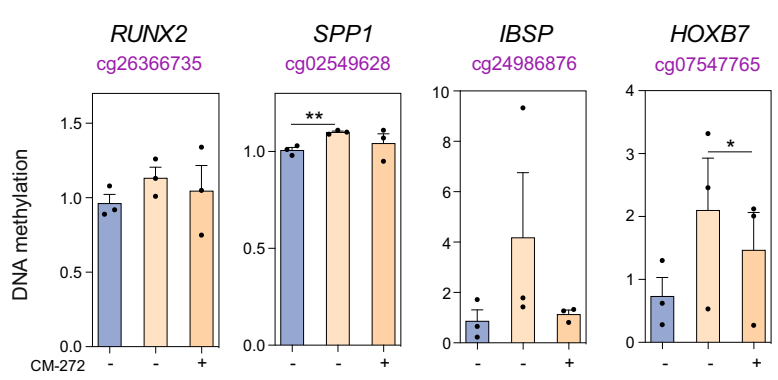

$\square$ MSC

$\mathrm{MSC}+\mathrm{MM} .1 \mathrm{~S}$

$\square$ MSC + RPMI-8226
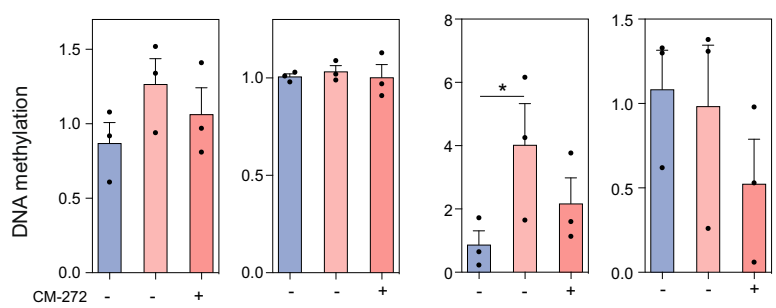

Fig. 5 CM-272 restores the gene expression levels and suppression of mineralization in MSCs from healthy donors exposed to MM cells. HD-MSCS (MSC; blue) were co-cultured with MM.1S (orange) or RPMI-8226 (red) cell lines separated by a transwell system in the presence (darker shade) or absence (lighter shade) of CM-272. A Relative expression and B DNA methylation of genes RUNX2, SPP1, IBSP, and HOXB7 was assessed. Gene expression was by normalization against RPL38. Bar plots represent mean \pm SEM of 3-5 independent experiments and statistical significance was calculated by paired one-tailed Student's $t$-test $\left({ }^{\star} p<0.05,{ }^{\star \star} p<0.01\right)$. C Mineralization was assessed by alizarin red staining in differentiated OBs from HD-MSCs co-cultured with MM.1S or RPMI-8226 cell lines in the presence or absence of CM-272. Representative micrographs show matrix mineralization by alizarin red staining of corresponding differentiated OBs. Data are represented as the mean \pm SEM from three independent experiments and paired two-tailed Student's $t$-tests were performed $\left({ }^{\star} p<0.05,{ }^{\star \star} p<0.01\right)$.

Bisulfite pyrosequencing. For total DNA extraction, cells were lysed using lysis buffer (50 mM Tris pH 8.8, $10 \mathrm{mM}$ EDTA pH 8.3, $100 \mathrm{mM} \mathrm{NaCl}, 1 \%$ SDS) in the presence of Proteinase K (Roche). Repeated centrifugation was performed to separate nucleic acids from lipids, in which DNA was subsequently precipitated using isopropanol and washed with $75 \%$ ethanol. 100-300 ng of isolated DNA were bisulfite (BS)-converted using EZ DNA Methylation-Gold ${ }^{\mathrm{m}}{ }^{\mathrm{T}}$ Kit (Zymo Research, CA, USA) according to manufacturers' instructions. BS-converted DNA ( 10 ng) was used as a template for amplification by conventional PCR using IMMOLA$\mathrm{SE}^{\mathrm{TM}}$ DNA Polymerase kit (Bioline, London, UK). PCR primers were designed with the PyroMark Assay Design v2.0.2 software (Qiagen). PCR products were pyrosequenced with the PyromarkTM Q24 system (Qiagen), according to the manufacturer's protocol.

Quantitative real-time PCR (qRT-PCR). Reverse transcription was performed using the Transcriptor First Strand cDNA Synthesis Kit (Roche) according to the manufacturer's instructions. qRT-PCR was performed using LightCycler ${ }^{\oplus} 480$ II System with LightCycler ${ }^{\oplus} 480$ SYBR Green Mix and data were analyzed with LightCycler ${ }^{\oplus} 480$ II Software, version 1.5, all provided by Roche. Reactions were performed in triplicate for both the target and the housekeeping gene ribosomal protein L38 (RPL38) used for normalization. Relative quantification of the target gene expression was calculated by the comparative threshold cycle $(\mathrm{Ct})$ method.

Co-culture system and MSC sorting. MSCs from healthy donors at passage $3(8 \times$ $10^{3}$ cells $\left./ \mathrm{cm}^{2}\right)$ or the hMSC-TERT cell line $\left(10 \times 10^{3}\right.$ cells $\left./ \mathrm{cm}^{2}\right)$ were first cultured in $100 \mathrm{~mm}$ culture dishes until they reached $\sim 85 \%$ confluency, and then MM.1S cells (1:3 MSC:MM.1S ratio) were added in RPMI 1640 medium supplemented with $10 \%$ FBS and antibiotics. MM cells were changed twice a week until day 14 when MSCs were recovered by trypsinization and flow cytometry-based sorting of $\mathrm{CD} 3^{+}$cells (BD Biosciences). For transwell experiments, MSCs from healthy donors were seeded on bottom chambers and MM.1S and RPMI-8226 cells were seeded on PET membrane inserts containing $1 \mu \mathrm{m}$ size pores to allow an exchange of soluble molecules. Transwell experiments were performed as in direct cocultures. For OB differentiation studies, MSCs from healthy donors were cultured in an osteogenic medium supplemented with $20 \%$ of conditioned media from the MM.1S cell line. This medium was changed twice a week until day 10 (ALP activity) or day 20 (OB mineralization). For the isolation of mouse MSCs, cells were stained with a combination of CD45-PE, Ter-119-PE, Sca-1-FITC, and PDFGRaAPC (BD Biosciences) as previously reported ${ }^{74}$ (Supplementary Fig. 5). Cell sorting 
A

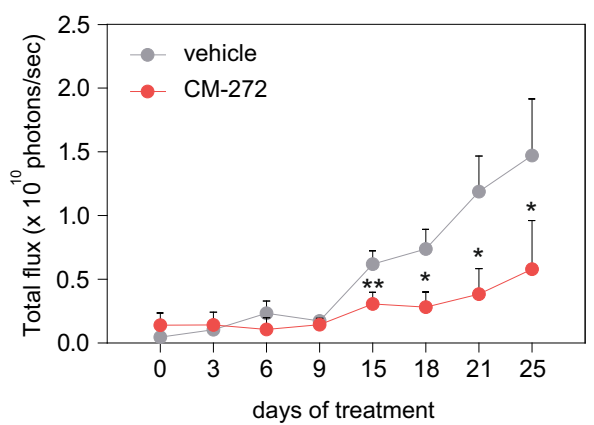

B

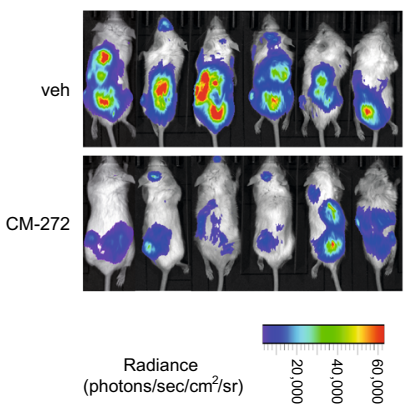

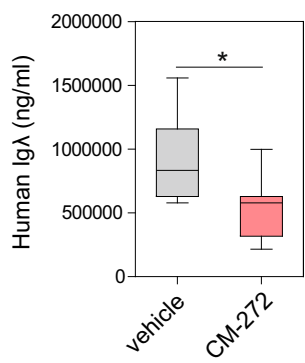

C

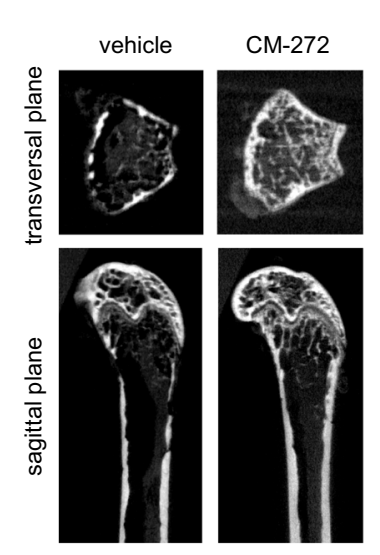

D

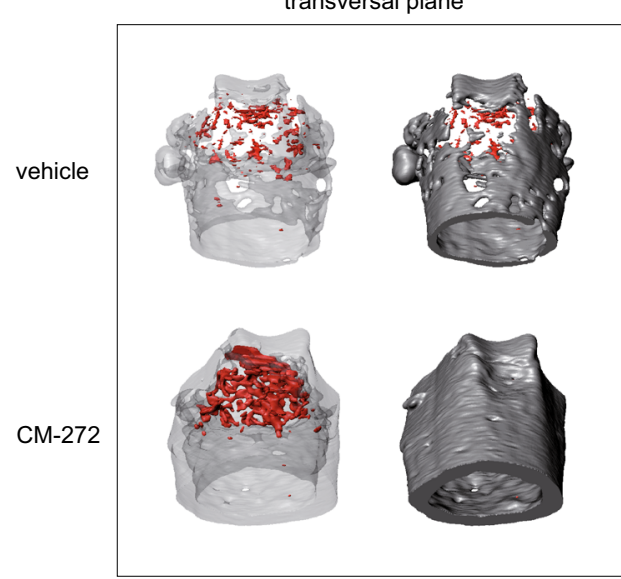

transversal plane

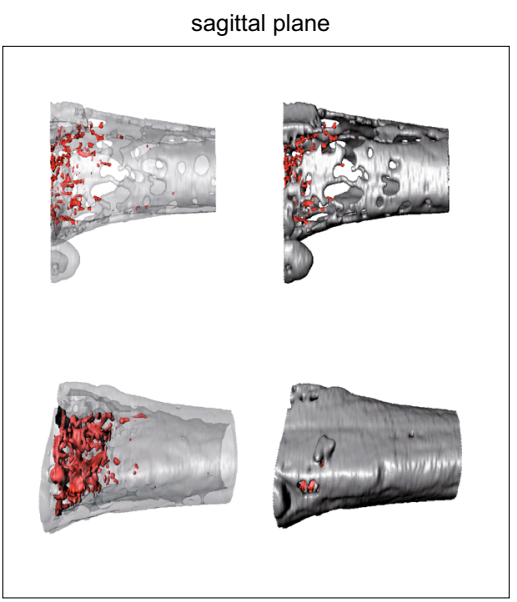

E

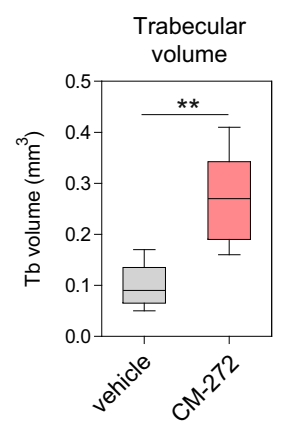

G

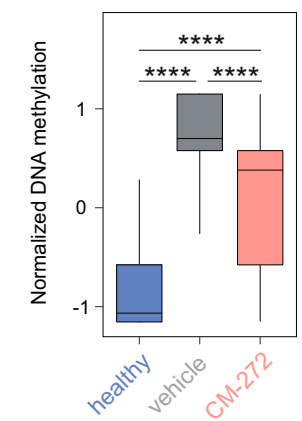

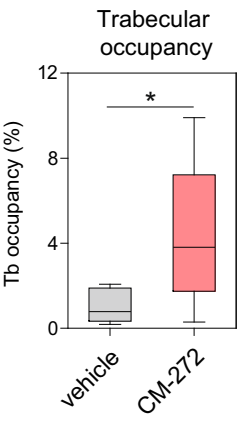

H
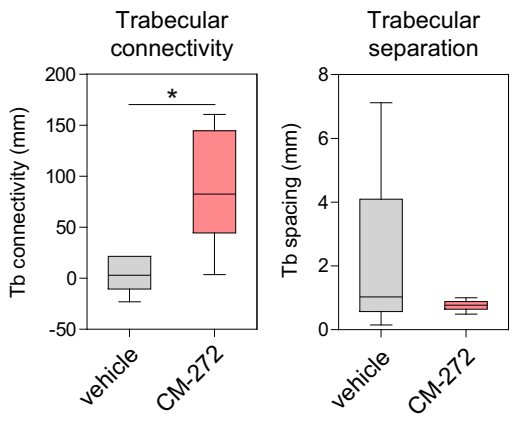

$\mathbf{F}$

\section{I}
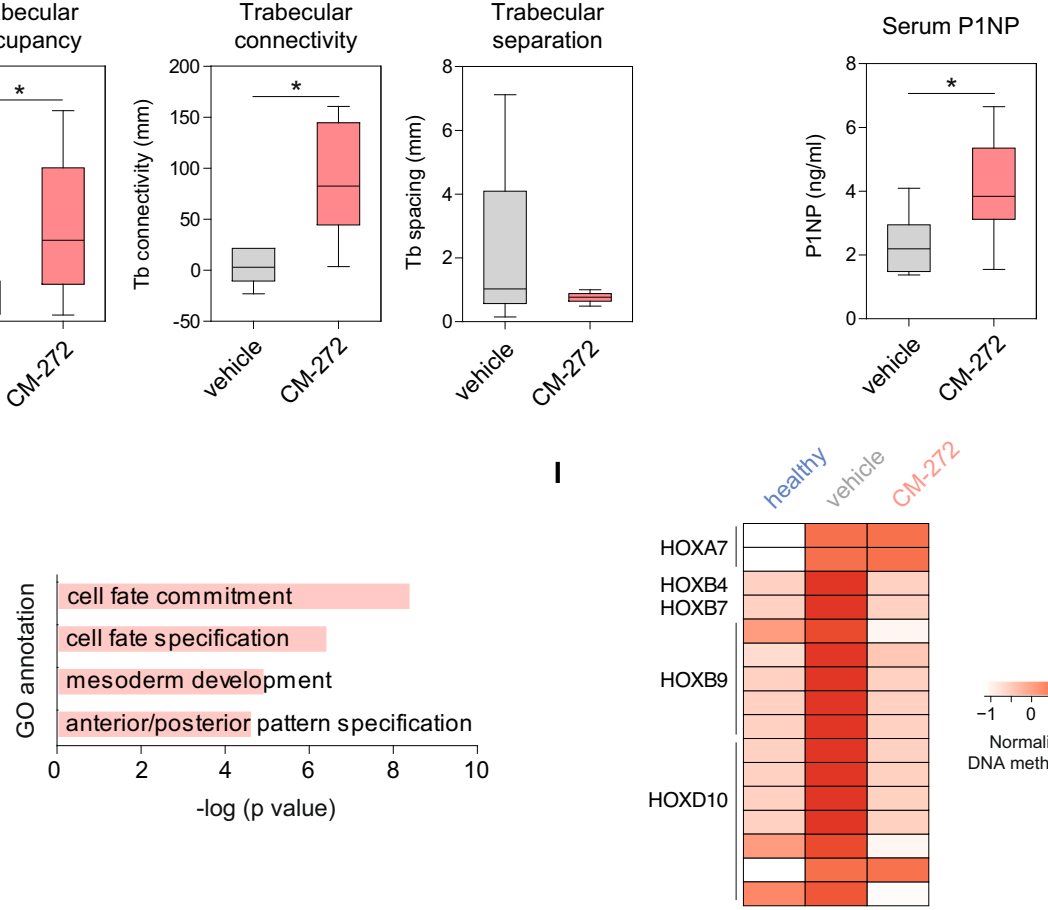

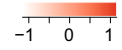

Normalized

DNA methylation experiments were performed by the Flow Cytometry Core Facility at Germans Trias i Pujol Research Institute utilizing FACSAria II cell sorter and analyzed using BD FACSDiva version 6.1.1 (BD Biosciences, San Jose CA).

OB differentiation assays. OBs were generated from mesenchymal precursors by culture in osteogenic medium (containing $5 \mathrm{mM} \beta$-glycerophosphate and $50 \mathrm{mg} / \mathrm{ml}$ ascorbic acid) and assayed as in Garcia-Gomez et al. ${ }^{75}$. Briefly, primary MSCs (P2-3) were cultured in osteogenic medium for the analysis of alkaline phosphatase (ALP) activity, expression of osteogenic markers (day 10), and formation of mineralized-nodules formation (day 20). ALP activity was determined by hydrolysis of $p$-nitrophenylphosphate (Sigma-Aldrich) into p-nitrophenol and NBT/ BCIP substrates (Roche), whereas mineralization was assessed by quantitative measurement of Alizarin Red (Sigma-Aldrich) staining and absorbance was 
Fig. 6 CM-272 prevents tumor-associated bone loss besides reducing multiple myeloma tumor burden. RPMI- 8226 -luc cells $\left(8 \times 10^{6}\right)$ were intravenously injected into NSG mice. After 4 weeks, mice were randomized into 2 groups [receiving vehicle (gray) and CM-272 (red); $n=6 /$ group] and treated for additional 4 weeks with dosing and regimen schedules as specified in Supplementary Methods. Tumor dissemination was checked by A bioluminescence measurement and $\mathbf{B}$ serum levels of human Ig $\lambda$ secreted by RPMI-8226-luc cells at specified time points. Line plots represent mean and SEM. Box plots represent median $\pm \mid \mathrm{QR}$, with whiskers representing the minimum and maximum. Statistical significance was determined utilizing paired two-tailed Student's $t$-test $\left({ }^{\star} p<0.05,{ }^{* *} p<0.01\right)$. C Representative microCT cross-sections at the metaphyses of distal femurs in a vehicle and CM-272treated mice in transversal (upper) and sagittal (down) planes. D Transversal (left) and sagittal (right) planes of corresponding 3D renderings from microCT images at distal femurs (trabecular bone in red, cortical bone in gray). E Trabecular bone morphometric parameters from microCT images were quantitated for trabecular bone volume, occupancy, connectivity, and separation. F Serum levels of the bone formation marker P1NP were quantified by ELISA. Graphs represent mean values \pm SEM with whiskers representing minimum and maximum values. CM-272-treated (red) mice were compared to the vehicle group (gray), where ${ }^{\star} p<0.05 ;{ }^{\star \star} p<0.01$ versus the vehicle control group. G Box plots showing DNA methylation levels of pooled MSCs obtained from healthy, vehicle- and CM-272-treated animals corresponding to hypermethylated CpGs between healthy and tumor-bearing animals. $\mathbf{H}$ GO enrichment analysis of CpG sites undergoing DNA hypermethylation changes in vehicle-treated MSCs versus MSCs from healthy mice. I Heatmap showing normalized DNA methylation levels of individual $\mathrm{CpGs}$ at selected Homeobox loci among animal groups. Data pooled from mice $(n=6)$ for each group with sufficient RRBS coverage ( $\geq 5$ valid sequencing reads per $\mathrm{CpG}$ ). $<0.01$ by paired two-tailed Student's $t$-test. G Box plots showing mean \pm SEM, with whiskers representing minimum and maximum values, of DNA methylation levels of pooled MSCs obtained from healthy (blue), vehicle- (gray), and CM272-treated (red) animals corresponding to hypermethylated $\mathrm{CpGs}$ between healthy and tumor-bearing animals. A paired two-tailed Student's $t$-test was performed to calculate statistical significance $\left.{ }^{\star \star \star \star} p<0.001\right)$. H GO enrichment analysis of $\mathrm{CpG}$ sites undergoing DNA hypermethylation changes in vehicle-treated MSCs versus MSCs from healthy mice. $p$-values were calculated utilizing a binomial test. I Heatmap showing normalized DNA methylation levels of individual $\mathrm{CpGs}$ at selected Homeobox loci among animal groups. The color scale ranges from white to red, representing low to high levels of DNA methylation. Data pooled from mice $(n=6)$ for each group with sufficient RRBS coverage ( $\geq 5$ valid sequencing reads per $C p G$ ).

measured by Multiskan Sky Microplate Spectrophotometer via SkanIt PC software (ThermoFisher).

MTT assay. MSCs were seeded in 96 well plates and treated with increasing concentrations of CM-272. MTT was added at a final concentration of $0.5 \mathrm{mg} / \mathrm{ml}$ and incubated at $37^{\circ} \mathrm{C}$ for $1 \mathrm{~h}$. Cells were then washed with PBS and incubated in the dark for $10 \mathrm{~min}$ in the presence of dimethyl sulphoxide. Absorbance at $570 \mathrm{~nm}$ was measured utilizing the Multiskan Sky Microplate Spectrophotometer.

Flow cytometry antibodies. Prior to analysis and sorting by flow cytometry, distinct amounts of MSCs were stained with fluorochrome-conjugated antibodies. Antibody specifications and concentrations used were the following: CD13-PE (BD Biosciences, 347406), CD45-PE (eBioscience, 12-0451-81), Ter-119-PE (eBioscience, 12-5921-81), Sca-1-FITC (eBioscience 11-5981-81), PDFGRa-APC (BD Biosciences, 562777), CD44-FITC (BD Biosciences, 347943), CD19-PerCP (BD Biosciences, 332780), CD90-FITC (BD Biosciences, 555595), HLA-DR-PerCP (BD Biosciences, 347402), CD14-FITC (BD Biosciences, 345784), CD166-PE (BD Biosciences, 559263), Cd45-PercCPcy5.5 (BD Biosciences, 332784), CD34-FITC (Invitrogen, 11-0349-42), CD73-PE (BD Biosciences, 550257), CD105-APC (R\&D System, FAB10971A).

Chromatin immunoprecipitation (ChIP)-quantitative PCR. MSCs $\left(15 \times 10^{3}\right.$ cells per IP) were cross-linked with $1 \%$ formaldehyde for $15 \mathrm{~min}$ and subjected to chromatin immunoprecipitation after sonication. ChIP-qPCR assays were performed using LowCell ChIP kit ${ }^{m}$ protein A (Diagenode) and the antibody $(5 \mu \mathrm{g})$ against H3K9me2 (H3K9me2 Abcam ChIP-grade, clone:mAbcam 1220, Ref: ab1220, Lot:GR45436-1). Data are represented as the ratio of the bound fraction over the input for each histone modification or factor. IgG was used as a negative control. Primer sequences were designed as close as possible from the $\mathrm{CpG}$ undergoing methylation changes. Primer sequences are shown in Supplementary Table 3. These experiments were performed with three biological replicates of each origin.

In vivo model. Animal experiments were conducted according to relevant ethical regulations for the use of laboratory animals and after acquired permission from the University of Salamanca Committee for animal experimentation (ref \# 0000061). BALB/c-Rag $2^{\text {null }} I L 2 r \gamma^{\text {null }}$ (BRG) mice // or NOD-scid IL2r $\gamma^{\text {null }}$ (NSG) mice were bred and maintained in the SPF area of the University of Salamanca Animal Facility with controlled environment conditions $\left(20-23^{\circ} \mathrm{C}, 12: 12\right.$ light/ dark cycles, $30-70 \%$ relative humidity) and fed ad libitum. CM-272 was solubilized in $0.9 \%$ saline solution. RPMI- 8226 -luc cells $\left(8 \times 10^{6}\right)$ were injected intravenously into 8-week-old NOD-SCID-IL-2R $\gamma^{-1-}$ (NSG) mice (Charles River Laboratories) and tumor development was monitored by noninvasive bioluminescence imaging (BLI) with a Xenogen IVIS 50 system (Caliper Life Sciences). After 4 weeks, animals were randomized into two groups ( $n=6$ /group) receiving vehicle ( $0.9 \%$ saline solution) or CM-272 (5 mg/kg, 5 times/week by intraperitoneal injection).

Microcomputed tomography analysis. One femur of each animal was fixed in $10 \%$ formalin in order to preserve bone microarchitecture. 3D X-ray tomographic images were acquired using a Quantum-GX microCT (Perkin Elmer) with the following parameters: $80 \mathrm{kVp}$ X-ray source voltage, $120 \mu \mathrm{A}$ current, and the highresolution scan protocol for a total acquisition time of $14 \mathrm{~min}$ and a gantry rotation of 360 degrees. The tomographic three-dimensional images containing the entire bone yielded a total of 512 slices, with isotropic 50 microns voxel size and a resolution of $512 \times 512$ pixels per slice. To perform the bone histomorphometry analysis a $(10 \times 10 \times 10 \mathrm{~mm})$ ROI containing the bone metaphysis was defined and subsequently reconstructed from the original scan at a resolution of 20 microns per voxel using the Quantum 3.0 software.

Analysis of trabecular microarchitecture in the distal femur was carried out using ImageJ v1.8.0 $0^{76}$. First of all, cortical and trabecular bones were segmented from the CT volume. To this end, the following steps were followed: (i) segmentation of the entire bone volume by thresholding the original volume to obtain a 3D binary mask; (ii) segmentation of empty volumes inside the cortical volume (trabecular-free zones) using logical operators over filled vs. unfilled versions of the result of step i; (iii) segmentation of the interior volume of the cortical bone by applying 10 morphological dilations followed by 10 morphological erosions to the 3D mask obtained in step ii; (iv) segmentation of the cortical bone by performing an XOR logical operation between the masks obtained in steps i and iii; and finally, (v) segmentation of the trabecular bone by performing an AND logical operation between the masks obtained in steps $i$ and iii. The final cortical and trabecular bone segmentations were further refined by applying a median filter to remove noise in the respective 3D masks. Cortical and trabecular bone volumes were then calculated by applying the segmentation masks on the original volume.

From the obtained trabecular masks, histomorphometry parameters were calculated using the BoneJ plugin (version 1.4.2) ${ }^{77}$. Finally, bone 3D reconstruction and visualization were performed using Amira 5.2 software (ThermoFisher Scientific).

ELISA. Serum levels of human $\operatorname{Ig} \lambda$ (indicating tumor burden) and N-terminal propeptide of type I procollagen (P1NP) (indicating bone formation) were measured in mice sera using the Human Lambda ELISA kit (Bethyl Laboratories, Texas, USA) and Rat/Mouse PINP EIA kit (Immunodiagnostic Systems, East Boldon, UK), respectively, following manufacturers' instructions. Absorbance was measured using the Multiskan Sky Microplate Spectrophotometer.

Reduced representation bisulfite sequencing (RRBS). Sorted MSCs from three groups (healthy, vehicle-treated, and CM-272-treated mice) were pooled in order to obtain a significant number of cells for performing the RRBS-seq. Isolated DNA from each pool was subjected to the RRBS pipeline as previously described ${ }^{78}$. In brief, purified DNA was digested with MspI and subjected to bisulfite conversion. Following PCR amplification, RRBS libraries were generated from sequenced DNA following previously published procedures (http://code.google.com/p/bsmap/ downloads/). Downstream normalization and analyses were performed following a previously published pipeline (http://rrbs-techdev.computational-epigenetics.org/). $\mathrm{CpG}$ annotation and GO enrichment analysis was performed utilizing the GREAT online tool.

Primers. All primers used are listed in Supplementary Table 3. 
Statistical analysis. Data are expressed as mean \pm SEM and the $n$ value for each in vitro assay is specified in the corresponding figure legend. Statistical analyses were carried out with Prism version 6.0 (GraphPad) and were performed using a two-tailed Mann-Whitney $U$ test or Student's $t$-test.

Reporting summary. Further information on research design is available in the Nature Research Reporting Summary linked to this article.

\section{Data availability}

The DNA methylation and expression data supporting the findings of this study have been deposited in the NCBI's Gene Expression Omnibus database under the accession code GSE137419. This SuperSeries (GSE137419) is composed of the following SubSeries: GSE137360 (methylation), GSE137369 (expression), and GSE137416 (methylation II). All the other data supporting the findings of this study are available within the article and its supplementary information files and from the corresponding author upon reasonable request. Source data are provided with this paper.

Received: 9 October 2019; Accepted: 14 December 2020; Published online: 18 January 2021

\section{References}

1. San-Miguel, J. F. \& Kantarjian, H. M. Multiple myeloma and chronic leukaemias in 2014: Improved understanding of disease biology and treatment. Nat. Rev. Clin. Oncol. 12, 71-72 (2014).

2. Kumar, S. K. et al. Multiple myeloma. Nat. Rev. Dis. Primers 3, 17046 (2017).

3. Raje, N. \& Roodman, G. D. Advances in the biology and treatment of bone disease in multiple myeloma. Clin. Cancer Res. 17, 1278-1286 (2011).

4. Saad, F. et al. Pathologic fractures correlate with reduced survival in patients with malignant bone disease. Cancer 110, 1860-1867 (2007).

5. Panaroni, C., Yee, A. J. \& Raje, N. S. Myeloma and bone disease. Curr. Osteoporos. Rep. 15, 483-498 (2017)

6. Dhodapkar, M. V. MGUS to myeloma: a mysterious gammopathy of underexplored significance. Blood 128, 2599-2606 (2016).

7. Sundararajan, S., Kumar, A., Korde, N. \& Agarwal, A. Smoldering multiple myeloma: emerging concepts and therapeutics. Curr. Hematol. Malig. Rep. 11, 102-110 (2016).

8. Amodio, N., D'Aquila, P., Passarino, G., Tassone, P. \& Bellizzi, D. Epigenetic modifications in Multiple Myeloma: recent advances on the role of DNA and histone methylation. Expert Opin. Ther. Targets 21, 91-101 (2016).

9. Das, R. et al. Microenvironment-dependent growth of preneoplastic and malignant plasma cells in humanized mice. Nat. Med. 22, 1351-1357 (2016).

10. Ghobrial, I. M., Detappe, A., Anderson, K. C. \& Steensma, D. P. The bonemarrow niche in MDS and MGUS: implications for AML and MM. Nat. Rev. Clin. Oncol. https://doi.org/10.1038/nrclinonc.2017.197 (2018).

11. Garcia-Gomez, A., Sanchez-Guijo, F., Del Cañizo, M. C., San Miguel, J. F. \& Garayoa, M. Multiple myeloma mesenchymal stromal cells: contribution to myeloma bone disease and therapeutics. World J. Stem Cells 6, 322-343 (2014).

12. Friedenstein, A. J., Petrakova, K. V., Kurolesova, A. I. \& Frolova, G. P. Heterotopic of bone marrow. Analysis of precursor cells for osteogenic and hematopoietic tissues. Transplantation 6, 230-247 (1968).

13. Corre, J. et al. Bone marrow mesenchymal stem cells are abnormal in multiple myeloma. Leukemia 21, 1079-1088 (2007).

14. $\mathrm{Xu}, \mathrm{S}$. et al. Impaired osteogenic differentiation of mesenchymal stem cells derived from multiple myeloma patients is associated with a blockade in the deactivation of the Notch signaling pathway. Leukemia 26, 2546-2549 (2012).

15. Reagan, M. R. \& Ghobrial, I. M. Multiple myeloma mesenchymal stem cells: characterization, origin, and tumor-promoting effects. Clin. Cancer Res. 18, 342-349 (2012).

16. Rajkumar, S. V. Multiple myeloma: 2016 update on diagnosis, riskstratification, and management. Am. J. Hematol. 91, 719-734 (2016).

17. Garayoa, M. et al. Mesenchymal stem cells from multiple myeloma patients display distinct genomic profile as compared with those from normal donors. Leukemia 23, 1515-1527 (2009).

18. Giuliani, N. et al. Bone osteoblastic and mesenchymal stromal cells lack primarily tumoral features in multiple myeloma patients. Leukemia $\mathbf{2 4}$, 1368-1370 (2010).

19. Todoerti, K. et al. Distinct transcriptional profiles characterize bone microenvironment mesenchymal cells rather than osteoblasts in relationship with multiple myeloma bone disease. Exp. Hematol. 38, 141-153 (2010).

20. Adamik, J. et al. EZH2 or HDAC1 inhibition reverses multiple myelomainduced epigenetic suppression of osteoblast differentiation. Mol. Cancer Res. 15, 405-417 (2017).
21. Goll, M. G. \& Bestor, T. H. Eukaryotic cytosine methyltransferases. Annu. Rev Biochem. 74, 481-514 (2005).

22. Cakouros, D. et al. Specific functions of TET1 and TET2 in regulating mesenchymal cell lineage determination. Epigenet. Chromatin 12, 3 (2019).

23. Garcia-Gomez, A., Rodríguez-Ubreva, J. \& Ballestar, E. Epigenetic interplay between immune, stromal and cancer cells in the tumor microenvironment Clin. Immunol. 196, 64-71 (2018).

24. Teschendorff, A. E. et al. Epigenetic variability in cells of normal cytology is associated with the risk of future morphological transformation. Genome Med. 4, 24 (2012)

25. Teschendorff, A. E. et al. The dynamics of DNA methylation covariation patterns in carcinogenesis. PLoS Comput. Biol. 10, e1003709 (2014).

26. Ernst, J. \& Kellis, M. ChromHMM: automating chromatin-state discovery and characterization. Nat. Methods 9, 215-216 (2012).

27. Heinz, S. et al. Simple combinations of lineage-determining transcription factors prime cis-regulatory elements required for macrophage and B cell identities. Mol. Cell 38, 576-589 (2010).

28. Ducy, P., Zhang, R., Geoffroy, V., Ridall, A. L. \& Karsenty, G. Osf2/Cbfa1: a transcriptional activator of osteoblast differentiation. Cell 89, 747-754 (1997).

29. Håkelien, A.-M. et al. The regulatory landscape of osteogenic differentiation. Stem Cells 32, 2780-2793 (2014).

30. Kanzler, B., Kuschert, S. J., Liu, Y. H. \& Mallo, M. Hoxa-2 restricts the chondrogenic domain and inhibits bone formation during development of the branchial area. Development 125, 2587-2597 (1998).

31. Jeong, B.-C. ATF3 mediates the inhibitory action of TNF- $\alpha$ on osteoblast differentiation through the JNK signaling pathway. Biochem. Biophys. Res. Commun. 499, 696-701 (2018).

32. Giuliani, N. et al. Myeloma cells block RUNX2/CBFA1 activity in human bone marrow osteoblast progenitors and inhibit osteoblast formation and differentiation. Blood 106, 2472-2483 (2005).

33. Seifert, A., Werheid, D. F., Knapp, S. M. \& Tobiasch, E. Role of Hox genes in stem cell differentiation. World J. Stem Cells 7, 583 (2015).

34. Verlinden, L. et al. Nrp2 deficiency leads to trabecular bone loss and is accompanied by enhanced osteoclast and reduced osteoblast numbers. Bone 55, 465-475 (2013).

35. Oshima, T. et al. Myeloma cells suppress bone formation by secreting a soluble Wnt inhibitor, sFRP-2. Blood 106, 3160-3165 (2005).

36. Kaneshiro, S. et al. Bruton tyrosine kinase (Btk) suppresses osteoblastic differentiation. J. Bone Miner. Metab. 33, 486-495 (2015).

37. Pizard, A. et al. Connexin 40 , a target of transcription factor Tbx5, patterns wrist, digits, and sternum. Mol. Cell. Biol. 25, 5073-5083 (2005).

38. Lanctôt, C., Moreau, A., Chamberland, M., Tremblay, M. L. \& Droui, J. Hindlimb patterning and mandible development require the Ptx1 gene. Development 126, 1805-1810 (1999).

39. Wei, X.-F., Chen, Q.-L., Fu, Y. \& Zhang, Q.-K. Wnt and BMP signaling pathways co-operatively induce the differentiation of multiple myeloma mesenchymal stem cells into osteoblasts by upregulating EMX2. J. Cell. Biochem. 120, 6515-6527 (2018).

40. Iyyanar, P. P. R. \& Nazarali, A. J. Hoxa2 inhibits bone morphogenetic protein signaling during osteogenic differentiation of the palatal mesenchyme. Front. Physiol. 8, 929 (2017).

41. Garcia-gomez, A. et al. Transcriptomic profile induced in bone marrow mesenchymal stromal cells after interaction with multiple myeloma cells: implications in myeloma progression and myeloma bone disease. Oncotarget 5, 8284-8305 (2014).

42. Estève, P.-O. et al. Direct interaction between DNMT1 and G9a coordinates DNA and histone methylation during replication. Genes Dev. 20, 3089-3103 (2006).

43. Tachibana, M., Matsumura, Y., Fukuda, M., Kimura, H. \& Shinkai, Y. G9a/ GLP complexes independently mediate H3K9 and DNA methylation to silence transcription. EMBO J. 27, 2681-2690 (2008).

44. San José-Enériz, E. et al. Discovery of first-in-class reversible dual small molecule inhibitors against G9a and DNMTs in hematological malignancies. Nat. Commun. 8, 15424 (2017)

45. Bárcena-Varela, M. et al. Dual targeting of histone methyltransferase G9a and DNA-methyltransferase 1 for the treatment of experimental hepatocellular carcinoma. Hepatology 69, 587-603 (2019).

46. Segovia, C. et al. Inhibition of a G9a/DNMT network triggers immunemediated bladder cancer regression. Nat. Med. 25, 1073-1081 (2019).

47. Rabal, O. et al. Discovery of reversible DNA methyltransferase and lysine methyltransferase G9a inhibitors with antitumoral in vivo efficacy. J. Med. Chem. 61, 6518-6545 (2018).

48. Xu, S., De Veirman, K., De Becker, A., Vanderkerken, K. \& Van Riet, I. Mesenchymal stem cells in multiple myeloma: a therapeutical tool or target? Leukemia 32, 1500-1514 (2018).

49. Kristinsson, S. Y. et al. Monoclonal gammopathy of undetermined significance and risk of skeletal fractures: a population-based study. Blood 116, 2651-2655 (2010). 
50. Capp, J.-P. \& Bataille, R. Multiple myeloma exemplifies a model of cancer based on tissue disruption as the initiator event. Front. Oncol. 8, 355 (2018).

51. Agirre, X. et al. Whole-epigenome analysis in multiple myeloma reveals DNA hypermethylation of B cell-specific enhancers. Genome Res. 25, 478-487 (2015).

52. Heuck, C. J. et al. Myeloma is characterized by stage-specific alterations in DNA methylation that occur early during myelomagenesis. J. Immunol. 190, 2966-2975 (2013).

53. Ledergor, G. et al. Single cell dissection of plasma cell heterogeneity in symptomatic and asymptomatic myeloma. Nat. Med. 24, 1867-1876 (2018).

54. van Nieuwenhuijzen, N., Spaan, I., Raymakers, R. \& Peperzak, V. From MGUS to multiple myeloma, a paradigm for clonal evolution of premalignant cells. Cancer Res. 78, 2449-2456 (2018).

55. da Silva, R. A. et al. HOXA cluster gene expression during osteoblast differentiation involves epigenetic control. Bone 125, 74-86 (2019).

56. Terpos, E., Ntanasis-Stathopoulos, I. \& Dimopoulos, M. A. Myeloma bone disease: from biology findings to treatment approaches. Blood 133, 1534-1539 (2019).

57. Raje, N. et al. Denosumab versus zoledronic acid in bone disease treatment of newly diagnosed multiple myeloma: an international, double-blind, doubledummy, randomised, controlled, phase 3 study. Lancet Oncol. 19, 370-381 (2018).

58. Zhou, W., Chen, H., Hong, X., Niu, X. \& Lu, Q. Knockdown of DNA methyltransferase-1 inhibits proliferation and derepresses tumor suppressor genes in myeloma cells. Oncol. Lett. 8, 2130-2134 (2014).

59. Das, D. S. et al. A novel hypoxia-selective epigenetic agent RRx-001 triggers apoptosis and overcomes drug resistance in multiple myeloma cells. Leukemia 30, 2187-2197 (2016).

60. Harada, T. et al. HDAC3 regulates DNMT1 expression in multiple myeloma: therapeutic implications. Leukemia 31, 2670-2677 (2017).

61. Yan, X. et al. 5-Azacytidine improves the osteogenic differentiation potential of aged human adipose-derived mesenchymal stem cells by DNA demethylation. PLoS ONE 9, e90846 (2014).

62. Rabal, O. et al. Detailed exploration around 4-aminoquinolines chemical space to navigate the lysine methyltransferase G9a and DNA methyltransferase biological spaces. J. Med. Chem. 61, 6546-6573 (2018).

63. Dominici, M. et al. Minimal criteria for defining multipotent mesenchymal stromal cells. The International Society for Cellular Therapy position statement. Cytotherapy 8, 315-317 (2006).

64. Aryee, M. J. et al. Minfi: A flexible and comprehensive Bioconductor package for the analysis of Infinium DNA methylation microarrays. Bioinformatics $\mathbf{3 0}$, 1363-1369 (2014).

65. Ritchie, M. E. et al. Limma powers differential expression analyses for RNAsequencing and microarray studies. Nucleic Acids Res. 43, e47 (2015)

66. Du, P., Kibbe, W. A. \& Lin, S. M. lumi: A pipeline for processing Illumina microarray. Bioinformatics 24, 1547-1548 (2008)

67. Hansen, K. D. IlluminaHumanMethylationEPICmanifest: manifest for Illumina's EPIC methylation arrays (2016).

68. Koch, C. M. et al. Pluripotent stem cells escape from senescenceassociated DNA methylation changes. Genome Res. 23, 248-259 (2013).

69. Teschendorff, A. E. et al. DNA methylation outliers in normal breast tissue identify field defects that are enriched in cancer. Nat. Commun. 7, 10478 (2016).

70. McLean, C. Y. et al. GREAT improves functional interpretation of cisregulatory regions. Nat. Biotechnol. 28, 495-501 (2010).

71. Lawrence, $M$. et al. Software for computing and annotating genomic ranges. PLoS Comput. Biol. 9, e1003118 (2013).

72. Carvalho, B. S. \& Irizarry, R. A. A framework for oligonucleotide microarray preprocessing. Bioinformatics 26, 2363-2367 (2010).

73. MacDonald, J. hugene10sttranscriptcluster.db: Affymetrix hugene10 annotation data (chip hugene10sttranscriptcluster) (2017).

74. Houlihan, D. D. et al. Isolation of mouse mesenchymal stem cells on the basis of expression of Sca-1 and PDGFR-a. Nat. Protoc. 7, 2103-2111 (2012).

75. Garcia-Gomez, A. et al. Dasatinib as a bone-modifying agent: anabolic and anti-resorptive effects. PLoS ONE 7, e34914 (2012).

76. Schneider, C. A., Rasband, W. S. \& Eliceiri, K. W. NIH Image to ImageJ: 25 years of image analysis. Nat. Methods 9, 671-675 (2012).

77. Doube, M. et al. BoneJ: free and extensible bone image analysis in ImageJ. Bone 47, 1076-1079 (2010).

78. Gu, H. et al. Preparation of reduced representation bisulfite sequencing libraries for genome-scale DNA methylation profiling. Nat. Protoc. 6, 468-481 (2011).

\section{Acknowledgements}

We thank CERCA Program/Generalitat de Catalunya and the Josep Carreras Foundation for institutional support. E.B. was funded by the Spanish Ministry of Science and Innovation (grant numbers SAF2014-55942-R and SAF2017-88086-R), co-funded by FEDER funds/European Regional Development Fund (ERDF) - a way to build Europe, and a Senior Research Award from the Multiple Myeloma Research Foundation (MMRF). C.O.-d.-S. was funded by the Spanish Ministry of Science, Innovation and Universities, under grant RTI2018-094494-B-C22 (MCIU/AEI/FEDER, UE). M.G. received financial support from the Spanish FIS-ISCIII (PI15/02156 and PI19/01384) and FEDER. A.G.G is funded by a postdoctoral contract of the Asociación Española Contra el Cáncer (AECC). F.P. was funded by grants from Instituto de Salud Carlos III (ISCIII), PI17/00701 and PI19/01352, TRASCAN (EPICA and Immunocell), Fundació La Marató de TV3, the Accelerator award CRUK/AIRC/AECC joint funder-partnership, CIBERONC (CB16/12/00489) and co-financed with FEDER funds and Fundación Ramón Areces (PREMAMM)

\section{Ethical declaration}

We have complied with all relevant ethical regulations for work with human participants in which informed written consent was obtained and the collection of samples and the study protocol for the use of those samples was approved by the Cancer Research Center-IBMCC Review Board (CICIC 2015/02156), the Clinical Ethics Committee for drug research in the Salamanca Health Area (CEIC 73/07/2015), the Clinical Research Committee of the Bellvitge University Hospital (ref. PR076/15) and the Research Ethics Committee at Universidad de Navarra (code 2017.218). Bone marrow samples were obtained conforming to ethical standards and good clinical practice for research work with principles expressed in the Declaration of Helsinki. All animal work was conducted according to relevant national and international guidelines for animal research and approved by the Bioethics Committee of the University of Salamanca (ref \# 0000061).

\section{Author contributions}

A.G.-G., T.L., J.R.-U., and E.B. conceived and designed experiments; A.G.-G., T.L., C.C.-F., J. R.-U., L.C., G.G.-T., and L.S.-S. performed experiments; A.G.-G., T.L., and F.C.-M. performed biocomputing analysis; M.M., L.S.-S., S.M., X.M., J.O., C.O.-d.-S., E.S.J.-E., M.E., X.A., F.P., and M.G. participated in the data acquisition (performed patient selection, provided drugs, samples, animals, and facilities); A.G.-G., T.L., J.R.-U., and E.B. analyzed and interpreted the data; A.G.-G., T.L., and E.B. wrote the paper. All authors read and approved the final manuscript.

\section{Competing interests}

The authors declare no competing interests.

\section{Additional information}

Supplementary information is available for this paper at https://doi.org/10.1038/s41467 020-20715-x.

Correspondence and requests for materials should be addressed to A.G.-G. or E.B.

Peer review information Nature Communications thanks Stuart Rushworth and the other, anonymous, reviewer(s) for their contribution to the peer review of this work.

Reprints and permission information is available at http://www.nature.com/reprints

Publisher's note Springer Nature remains neutral with regard to jurisdictional claims in published maps and institutional affiliations.

Open Access This article is licensed under a Creative Commons Attribution 4.0 International License, which permits use, sharing, adaptation, distribution and reproduction in any medium or format, as long as you give appropriate credit to the original author(s) and the source, provide a link to the Creative Commons license, and indicate if changes were made. The images or other third party material in this article are included in the article's Creative Commons license, unless indicated otherwise in a credit line to the material. If material is not included in the article's Creative Commons license and your intended use is not permitted by statutory regulation or exceeds the permitted use, you will need to obtain permission directly from the copyright holder. To view a copy of this license, visit http://creativecommons.org/ licenses/by/4.0/

(C) The Author(s) 2021 